\title{
Inventaire des moustiques (Diptera : Culicidae) des îles du sud-ouest de l'océan Indien, Madagascar excepté — Une revue critique
}

\author{
Philippe Boussès, Gilbert Le Goff \& Vincent Robert \\ UMR MIVEGEC (Maladies Infectieuses et Vecteurs : Écologie, Génétique et Évolution), IRD, CNRS, \\ Université de Montpellier, Montpellier, France \\ philippe.bousses@ird.fr \\ gilbert.legoff@ird.fr \\ Corresponding author: vincent.robert@ird.fr
}

\begin{abstract}
Summary. Inventory of the mosquitoes (Diptera: Culicidae) of the islands of southwestern Indian Ocean, Madagascar excluded-A Critical Review. The biodiversity of mosquitoes in the islands of southwestern Indian Ocean is the concern of numerous publications. Here, we propose a synthetic inventory and the analysis of the mosquito diversity, based on the available literature. A comprehensive annotated checklist of mosquito species has been recently published on Madagascar; reason why this land is excluded of our work. Studied area encompasses 28 tropical islands in the southern hemisphere: 4 islands in the Comoros archipelago, 5 Scattered Islands (îles Éparses), 5 in Mascarene, 11 in the Seychelles and 3 in the Chagos archipelago. In total, the mosquito list present 73 valid species, of which 10 Anophelinae and 63 Culicinae. The number of species that are distributed in these islands only is 20 , i.e. $27 \%$, which is a remarkable level for endemism. The richness in mosquito species in these islands is analysed through several aspects including geography, local speciation and natural or human dissemination. This updated inventory increases by $33 \%$ the number of known species by regard to the previous inventory published by Julvez \& Mouchet in 1994. The historical responsibility of Humans in the introduction of new mosquito species in these islands is strongly documented. For instance, the species with the highest distribution among islands are Aedes aegypti, Ae. albopictus and Culex quinquefasciatus. The islands belong to the afrotropical biogeographic area and, logically, the majority (63\%) of mosquito species present phylogenetic affinities with continental Africa and/or Madagascar; interestingly, the number of species present in these islands and in Madagascar but absent in continental Africa is higher than the number of species present in these islands and in continental Africa but absent in Madagascar (respectively 12 and 2 species). Thanks to valuable increase in the sampling effort, our knowledge of the culicidian fauna is increasing in these islands that constitute indisputably hotspots of biodiversity.
\end{abstract}

Keywords: biodiversity, faunistics, endemism, distribution, origin of populations

Résumé. La biodiversité des moustiques dans les îles du sud-ouest de l'océan Indien a fait l'objet de nombreuses publications. Nous proposons ici un inventaire des espèces et une analyse des peuplements, en se basant sur les données disponibles dans la littérature. Madagascar est exclu de cette étude car un inventaire des espèces de moustiques vient d'y être réalisé. La présente étude retient 28 îles tropicales de l'hémisphère sud : 4 îles dans 1'archipel des Comores, 5 îles Éparses, 5 aux Mascareignes, 11 aux Seychelles et 3 dans l'archipel des Chagos. Au total, 73 espèces valides de moustiques ont été recensées, dont 10 Anophelinae et 63 Culicinae. Le nombre d'espèces exclusivement présentes dans ces îles est de 20 , soit un remarquable endémisme concernant $27 \%$ des espèces. La richesse spécifique est analysée sous plusieurs aspects incluant la géographie, la spéciation sur place, la dissémination des populations par des voies naturelles ou humaines. Le présent inventaire accroit de $33 \%$ le nombre d'espèces connues par rapport à l'inventaire précédemment publié par Julvez \& Mouchet en 1994.

Mots-clés : Culicidae, océan Indien, île, biodiversité, endémisme, distribution, origine du peuplement 


\section{Introduction}

Les îles du sud-ouest de l'océan Indien (SOOI) ont attiré l'attention cette dernière décennie en raison des épidémies de dengue, de chikungunya et de fièvre de la Vallée du Rift dont les agents pathogènes sont des arbovirus essentiellement transmis par des moustiques Aedini (Zeller 1998 ; Clements 2012). Ce vaste complexe insulaire s'étend sur un large espace d'environ $5000 \mathrm{~km}$ du nord au sud entre l'équateur et le tropique du Capricorne et de $5000 \mathrm{~km}$ d'est en ouest, à l'est du continent africain. Par le fait que les moustiques sont des organismes terrestres, leur peuplement est ici contraint par l'existence d'îles plus ou moins distantes les unes des autres, et dont la plupart sont fort éloignées de tout continent. La fragmentation de l'espace entre les îles constitue un frein évident à la dissémination pour les moustiques, insectes peu enclins à franchir par eux-mêmes les distances inter-îles qui sont considérables et qui excèdent de beaucoup leur capacité de vol autonome. Le régime des vents et des courants intervient en paramètre essentiel pour la compréhension de la biogéographie insulaire, tout particulièrement pour les insectes (Paulian 1961; Julvez \& Mouchet 1994). Les vents dominants tels que les alizés du sud-est et la manifestation récurrente de cyclones ont certainement favorisé le transport passif de ces insectes, contribuant pour partie au peuplement des îles. Mais le vent n'est pas le seul facteur favorisant la dispersion des espèces dans cet espace fragmenté. Il a largement été démontré que le caractère anthropophile de nombreux moustiques était positivement associé à leur dissémination (Julvez \& Mouchet 1994 ; Service 1997), si bien que l'homme est ainsi à la fois acteur et victime des introductions d'espèces de moustiques, tant pour les aspects de nuisances dus aux piqûres, que pour les aspects de transmission vectorielle de nombreux agents pathogènes. Ces territoires insulaires constituent un terrain d'étude favorable pour la compréhension des mécanismes qui gouvernent la présence/absence d'une espèce dans tel ou tel habitat.

Trois synthèses de la biodiversité culicidienne des îles du SOOI ont été publiées au $20^{\mathrm{e}}$ siècle par Mattingly \& Brown (1955), Grjebine (1966) puis Julvez \& Mouchet (1994). Cette dernière recensait 55 espèces. Depuis, de nouvelles prospections ont été menées sur les îles Éparses (Girod \& Le Goff 2005 ; Bagny et al. 2009b), aux Seychelles (Le Goff et al. 2012), aux Comores (Marsden et al. 2013), à Mayotte (Le Goff et al. 2013, 2014) et à la Réunion (Girod 2001 ; Boussès et al. 2013), et ont suscité la découverte d'espèces nouvelles pour la zone ou même pour la science. Le présent article se propose de synthétiser toutes ces études disponibles pour faire un état de la biodiversité culicidienne des îles du SOOI. De par leurs caractéristiques géographiques, ces différentes îles offrent aux moustiques des opportunités d'installation très différentes.

\section{Matériel et méthodes \\ Zone géographique considérée}

Le présent article prend en compte toutes les îles du canal du Mozambique et de l'océan Indien au sud de l'équateur et dans la moitié ouest de l'océan Indien (Figure 1). Les îles retenues sont finalement toutes tropicales et au nord du tropique du Capricorne. Elles relèvent sur le plan biogéographique de la région éthiopienne et, pour nombre d'entre elles, de la sous-région malgache (Paulian 1961).

Le présent article ne mentionnera pas les trois îles tanzaniennes de l'archipel de Zanzibar (Pemba, Unguja et Mafia), trop proches du continent africain et pour lesquelles un inventaire récent des espèces de moustiques n'est à notre connaissance pas disponible. Ni les îles les plus australes situées au-delà du tropique du Capricorne et même au sud du $35^{\mathrm{e}}$ parallèle sud : les îles Saint-Paul et Nouvelle-Amsterdam, les îles Marion et Prince Edouard, les îles de l'archipel Crozet, les îles Kerguelen, les îles Heard-etMacDonald; dans ces îles australes exposées aux vents dans la zone des $40^{\mathrm{e}}$ rugissants, la faune entomologique est très pauvre et les moustiques en sont absents (Eaton 1879; Chapuis et al. 2002 ; Evenhuis 2007).

Le présent article ne considère pas Madagascar pour deux raisons. La première est qu'une synthèse exhaustive de la biodiversité culicidienne a été achevée récemment (Tantely et al. 2016), dénombrant 237 espèces et un taux d'endémisme de $59 \%$. Cette synthèse sera largement utilisée dans la discussion du présent article. La seconde raison est que de nombreux auteurs considèrent Madagascar comme un sous-continent plutôt que comme une île (Paulian 1961). Avec $585000 \mathrm{~km}^{2}$, soit une surface 233 fois plus étendue que la plus grande île de la région (Réunion), Madagascar forme à lui seul la sous-région biogéographique malgache. Les mécanismes de l'évolution y ont fonctionné de façon relativement autonome sous l'influence de l'isolement prolongé des terres émergées et de la diversité des biotopes 
qui ont favorisé une spéciation intense de la faune culicidienne. De ce fait, la comparaison entre Madagascar et les îles du SOOI pourrait être d'une pertinence discutable.

La plupart des îles de l'océan Indien sont apparues entre le Miocène et le début du Pléistocène et sont la résultante d'une activité volcanique. Seules les îles granitiques des Seychelles sont nettement plus anciennes, formées il y a $700 \mathrm{Ma}$ (millions d'années) à partir de fragments émergés du socle Précambrien (Michot \& Deutsch 1977).

\section{Niveau taxonomique considéré}

Le présent article se focalise sur le niveau taxonomique de l'espèce et ne prend pas en compte les niveaux inférieurs (tels que les sous-espèces).

\section{Sources bibliographiques}

Les informations concernant les moustiques (distribution, bio-écologie, rôle vecteur, etc.) rapportées ici proviennent essentiellement de données disponibles dans la littérature scientifique classique (livres, articles) ou dans la littérature grise. Toute la littérature grise (documents internes, rapports d'activité, rapports de mission...) mentionnée dans la bibliographie du présent article est disponible sur le site http://horizon.documentation.ird.fr ou à l'adresse https://arim.ird.fr/arim/docus. Quelques informations ponctuelles découlent de spécimens de la zone d'étude conservés dans la collection ARIM de l'IRD Montpellier, France (https://arim.ird.fr). L'avis des auteurs, lorsqu'il est exprimé, l'est de façon explicite. Il résulte de cette façon de procéder que les petites îles et îlots, très nombreux dans la zone géographique considérée, ne sont pas pris en compte, faute de données disponibles relatives aux moustiques.

Les premières références bibliographiques retenues datent du début du $20^{\mathrm{e}}$ siècle, les dernières de 2017. Les résultats de présence/absence des espèces culicidiennes concernent essentiellement la situation actuelle. Lorsqu'une espèce culicidienne a été observée dans une île par le passé, mais est actuellement considérée comme disparue, elle n'est donc pas comptabilisée dans les tableaux ci-dessous.

\section{Nomenclature}

Nous utiliserons la taxonomie, la nomenclature ainsi que les abréviations recommandées par Wilkerson et al. (2015). Cette taxonomie n'étant pas acceptée par tous les spécialistes (Reinert et al. 2009), nous indiquons la correspondance avec la taxonomie retenue pour la tribu des Aedini sur le site www.mosquito-taxonomic-inventory (Tableau 1).

\section{Difficultés taxonomiques d'identification des espèces culicidiennes}

Dans l'étude de la biodiversité des moustiques ici présentée, de nombreuses difficultés concernent plusieurs taxa, en particulier à propos de leur statut spécifique ou sub-spécifique, d'éventuelles erreurs d'identification entre plusieurs espèces morphologiquement proches, et enfin des mises en synonymie. Elles sont ici listées et, pour la plupart d'entre elles, explicitées.

Quatre taxa collectés sur diverses îles du SOOI demeurent actuellement indéterminés au niveau de l'espèce. Deux correspondent à des espèces nouvelles non formellement nommées : Aedes sp. A d'Aldabra (Le Goff et al. 2012) et Culex sp. A de Mayotte (Le Goff et al. 2014). Les deux autres taxa, observés sur Anjouan, sont notés "unknown" au niveau de l'espèce : il s'agit d'une unique femelle Anopheles (Table S6 in Marsden et al. 2013) et d'un Culex collecté dans un ruisseau (Table S5 in Marsden et al. 2013) dont on ignore s'il correspond ou non à $C x$. sp. A mentionné ci-dessus. Les deux premières de ces quatre espèces (Ae. sp. A et $C x$. sp. A) sont retenues dans le présent article car elles sont identifiables, au moins au stade larvaire, puisqu'elles ont été partiellement décrites. Contrairement aux deux dernières (Anopheles "unknown" et Culex "unknown"), non retenues dans le présent article pour des raisons opposées, puisqu'aucune information morpho-taxonomique n'est disponible.

Deux espèces considérées comme endémiques ont été récoltées à de très rares occasions et à de très rares exemplaires. La première est Anopheles comorensis Brunhes, 1997, qui a été décrite sur une seule femelle capturée sur homme, le 5 mars 1971 dans le village de Sada sur l'île de Mayotte (Brunhes et al. 1997). Elle n'a jamais été retrouvée depuis et en conséquence tous les autres stades restent inconnus. La seconde espèce est Ae. vinsoni Mattingly, 1953, considérée endémique de Maurice et décrite à partir d'une seule femelle capturée par J. Vinson à Moka, le 30 mai 1946. Une seconde femelle a été capturée à Eau Bleue, le 7 juillet 1961 (Mattingly 1963). De par sa ligne longitudinale blanche sur le scutum, ce 
taxon évoque Aedes albopictus (Skuse, 1894). D'ailleurs, Mattingly (1967) émet l'hypothèse que ce taxon résulte d'une hybridation entre Ae. albopictus et Ae. mascarensis. Aucune autre capture n'a été signalée depuis et tous les autres stades restent inconnus. Au cours des prochaines décennies, si aucun nouveau spécimen de ces espèces n'est collecté, l'extinction de ces deux espèces devra être discutée.

Culex comorensis Brunhes, 1977 comporte deux sous-espèces. La sous-espèce nominative $C x . c$. comorensis est connue de Mohéli, Anjouan, Mayotte et Madagascar, alors que la sous-espèce $C x . c$. kartalae Brunhes, 1977 est seulement connue de Grande Comore. Ces deux sous-espèces sont morphologiquement semblables au niveau des imagos femelles et de la nymphe ; seuls des détails des genitalia mâles et du stade larvaire permettent de les distinguer. Marsden et al. (2013) n'ont pas mentionné la sous-espèce observée tant à Grande Comore que sur les autres îles pourtant prospectées au niveau des gîtes larvaires.

Aedes mascarensis MacGregor, 1924 est morphologiquement proche d'Ae. aegypti (Linnaeus, 1762) mais il existe des différences comportementales et morphologiques marquées suffisantes pour en faire deux espèces distinctes (McClelland 1967), même si ces deux taxa sont interféconds en laboratoire (Hartberg \& Craig 1968, 1970). Leurs genitalia sont très semblables mais le gonostyle permet de les différencier (Huang 2004). Delatte et al. (2011), se basant sur l'ADNmt, suggèrent que ce taxon est une sous-espèce, voire une forme d'Ae. aegypti alors que Gloria-Soria et al. (2016) prennent fermement le parti de considérer Ae. mascarensis comme une espèce valide, génétiquement sœur mais bien distincte d'Ae. aegypti.

Aedes simpsoni Theobald, 1905 a été cité comme présent aux Comores par plusieurs auteurs. Le groupe Simpsoni est composé de trois espèces (Ae. simpsoni, Ae. bromeliae et Ae. lilii) qui sont délicates à distinguer. Nous pensons que la mention d'Ae. simpsoni aux Comores est fautive et correspond en fait à Ae. bromeliae qui, sur la base de critères morphologiques et génétiques, est la seule espèce de ce complexe présente à Mayotte (Le Goff et al. 2013) et sur l'archipel des Comores. Un argument supplémentaire concerne l'aire de répartition d'Ae. simpsoni, restreinte à l'Afrique continentale australe (Afrique du Sud et Swaziland).

Une autre difficulté concerne le complexe d'espèce $C x$. pipiens s.l. Le taxon $C x$. pipiens a été rapporté à Maurice (Mac Gregor 1923). Nous pensons qu'il faut interpréter ces mentions de Cx. pipiens comme $C x$. pipiens s.l. car il est très probable que ce soit ici le vicariant tropical du complexe, $C x$. quinquefasciatus Say, 1823, ubiquiste et abondant dans toute la zone, qui est ici observé. En effet, $C x$. pipiens n'a jamais été certifié dans ces îles et la distinction morphologique entre les femelles des deux espèces est très hasardeuse.

La présence de $C x$. univittatus Theobald, 1901, tant à Maurice qu'à Mohéli (Comores), reste à confirmer car cette espèce peut facilement être confondue avec $C x$. neavei Theobald, 1906. Ces deux espèces relèvent du groupe Pipiens et du sous-groupe Univittatus. La présence exclusive de $C x$. neavei à la Réunion a été établie sur des arguments morphométriques et génétiques (Boussès et al. 2013). Nous pensons qu'une telle analyse devrait être menée sur des spécimens de Maurice et de Mohéli pour trancher sur leur statut spécifique respectif, sachant que leur capacité vectorielle respective diverge (Jupp et al. 1986).

Il y a eu, par le passé, une confusion concernant Ae. fryeri (Theobald, 1912) et l'espèce Aedes sp. A. Le stade larvaire correspondant à Ae. fryeri a été brièvement décrit par Mattingly (1963) sous le nom d'Aedes mombasaensis, espèce qui fut placée en synonymie de Ae. fryeri par van Someren (1972). Hopkins (1952) a décrit la larve d'Aedes sp. A de l'atoll d'Aldabra et l'a attribuée à tort à l'espèce Ae. fryeri, comme nous avons pu l'établir sur la base de séquences nucléotidiques (Le Goff et al. 2012). L'adulte d'Aedes sp. A reste à décrire.

Aedes dufouri (Hamon, 1953) aurait été capturé uniquement au stade larvaire en 2008 sur Europa (Bagny et al. 2009b). Or, à ce stade de développement, Ae. dufouri est morphologiquement très proche d'Ae. fryeri, seule espèce capturée dans le même temps à l'état adulte sur l'île. Une comparaison de séquence partielle de l'ADN 28S du rDNA de divers spécimens identifiés comme Ae. dufouri issus de la collection ARIM, IRD Montpellier, France, provenant tant de la Réunion que de l'île Europa ou de Madagascar, donne une divergence génétique significative variant de $6 \%$ à $14 \%$ entre les spécimens de l'île de la Réunion et ceux provenant de l'île Europa et de Madagascar. Cette divergence est suffisante pour confirmer le caractère endémique d'Ae. dufouri dans la seule île de la Réunion. En accord avec Girod \& Le Goff (2005) et Boussès et al. (2013), nous estimons que Ae. dufouri est absent de l'île Europa. 
Aedes seychellensis (Theobald, 1912) a été mis en synonymie d'Ae. albocephalus (Theobald, 1903) sur des bases génétiques utilisant des portions de séquences des gènes NDH4 and ITS2 (Le Goff et al. 2012). Aedes brunhesi Reinert, 1999, espèce endémique de Mayotte et décrite à partir de deux mâles uniquement, est extrêmement difficile à différencier d'Ae. monetus Edwards, 1935 d'origine malgache. En effet, certains critères morphologiques considérés comme discriminants ne sont pas constants. L'analyse à l'aide de plusieurs marqueurs moléculaires permettra peut-être à l'avenir de statuer sur l'existence ou non de ces deux taxa.

Deux espèces de Culex du sous-genre Eumelanomyia Theobald, 1909 sont difficiles à identifier, $C x$. sunyaniensis Edwards, 1941 et Cx. wigglesworthi Edwards, 1941. La première est décrite du Ghana (et présente au Mozambique et à Madagascar-Vaingandrano) et la seconde est décrite du Sierra Leone (et présente à Mayotte et Madagascar-Manakara) (Mardsen et al. 2013 ; Le Goff et al. 2014 ; Tantely et al. 2016). Ce sous-genre comprend six espèces valides dans la zone d'étude dont quatre ont une origine afrotropicale, une a une origine malgache et une est endémique aux Seychelles. Les espèces d'origine afrotropicale ont été décrites d'Afrique Centrale (Cx. insignis) et d'Afrique de l'Ouest (Cx. horridus, $C x$. sunyaniensis, $C x$. wigglesworthi) si bien qu'on peut se poser la question de l'existence de vraies communautés de reproduction (i.e. des espèces) entre ces zones d'Afrique continentale et les îles du SOOI. L'analyse moléculaire comparative des populations des diverses sous-régions biogéographiques permettra peut-être à l'avenir de clarifier leur statut taxonomique respectif.

Enfin, des difficultés d'identification concernent certaines espèces du genre Orthopodomyia Theobald, 1904 car, en dépit du travail de synthèse fait sur ce genre (Brunhes \& Hervy 1995), la différenciation entre deux taxa proches reste parfois problématique. C'est le cas entre les espèces Or. comorensis et Or. joyoni dans l'archipel des Comores (Le Goff et al. 2014). En ce qui concerne Or. reunionensis, endémique de la Réunion, et Or. arboricollis, endémique de l'île Maurice (Boussès et al. 2013), les seuls critères morphologiques sont ici inopérants pour définitivement statuer sur la validité du statut spécifique de ces deux taxa.

\section{Résultats}

L'analyse bibliographique a retenu 36 références, dont 30 ont fait l'objet d'une prospection sur le terrain (Tableau S1 feuille 2). Les îles sont présentées ci-après par regroupement géographique, dans le canal du Mozambique puis dans l'océan Indien. Au total, 28 îles ou groupes d'îles ont été retenus : 4 dans 1'archipel des Comores, 5 îles Éparses, 5 relevant des Mascareignes, 5 dans les Seychelles granitiques, 6 dans les Seychelles coralliennes et 3 dans l'archipel des Chagos. Dans ces îles, le nombre total d'espèces culicidiennes est de 73 (Tableau S1 feuille 1).

\section{L'archipel des Comores}

Cet archipel est situé dans la partie nord du canal du Mozambique, à peu près à égale distance de Madagascar et du continent africain. Il est composé de quatre îles volcaniques positionnées dans un alignement nord-ouest sud-est, par ordre d'ancienneté croissante : Grande Comore, Mohéli, Anjouan et Mayotte. Au plus proche, ces îles sont situées à $280 \mathrm{~km}$ du continent africain et à $320 \mathrm{~km}$ de Madagascar; elles sont distantes entre elles de 40 à $80 \mathrm{~km}$. Elles sont soumises à des conditions climatiques similaires, de type tropical humide, avec une saison chaude et pluvieuse s'étendant de décembre à fin mars, quoiqu'il y ait des variations locales de la pluviométrie liées à l'altitude.

La faune culicidienne des îles de Grande Comore, Anjouan et Mohéli a fait l'objet de prospections sommaires à l'exemple de celles effectuées par Brygoo \& Escolivet (1955), Grjebine (1956a, 1956b), Chauvet (1967), Prod'hon (1972) ou Sabatinelli et al. (1994) dans le cadre d'expertises sur les vecteurs des parasites du paludisme ou de la filariose. L'étude la plus complète dans l'archipel fut celle de Brunhes (1978) qui ajouta 22 espèces aux 17 espèces connues jusqu'alors. Un travail récent (Marsden et al. 2013) a permis la récolte de 21 espèces dans les trois îles Grande Comore, Anjouan et Mohéli. Enfin Mayotte a fait dernièrement l'objet d'une prospection minutieuse (Le Goff et al. 2014) qui rassembla 40 espèces, dont 8 nouvelles pour l'île.

Au bilan, ce sont 52 espèces qui ont colonisé une ou plusieurs îles de cet archipel (Tableau 2). Seules 13 d'entre-elles sont toutefois présentes sur l'ensemble des quatre îles. Il s'agit d'Ae. aegypti, Ae. bromeliae, Ae. vittatus, Anopheles gambiae, An. merus, An. pretoriensis, Cx. comorensis, Cx. decens, Cx. quinquefasciatus, Cx. simpsoni, Lutzia tigripes, Eretmapodites quinquevittatus, Er. subsimplicipes. 
A signaler qu'An. squamosus a été mentionné dans l'archipel des Comores (Brygoo \& Escolivet 1955), mention unique que nous considérons comme trop douteuse pour être ici prise en compte.

Grande Comore. Cette île est la plus vaste $\left(1148 \mathrm{~km}^{2}\right)$, la plus jeune $(0,13 \mathrm{Ma})$, la plus élevée aussi (mont Karthala $2361 \mathrm{~m}$ ). Elle héberge actuellement une population de 400000 habitants, essentiellement rurale. Son volcanisme est encore actif et son sol, peu décomposé, est très perméable : malgré de fortes précipitations, il ne permet habituellement pas le maintien d'eau de surface. Les seuls gîtes larvaires possibles sont donc les phytotelmes et les gîtes anthropiques tels que les nombreuses citernes et bassins d'ablutions, les petits containers ou les pirogues abandonnées. Les autres gîtes larvaires sont temporaires et rares (trous de rocher, flaques, petites mares). Au début du $20^{\mathrm{e}}$ siècle, Grande Comore était réputée pour être indemne de paludisme (Julvez \& Blanchy 1988). Mais une première épidémie s'est produite dans cette île en 1920 ; elle était manifestement postérieure à l'introduction et l'implantation de vecteurs qui se développaient dans l'eau contenue dans les nombreuses citernes villageoises à ciel ouvert (Brunhes 1978). Anopheles funestus n'a jamais été collecté à Grande Comore et le seul vecteur de paludisme connu est An. gambiae s.s. Les prospections entomologiques ont été peu nombreuses sur cette île, toutefois 19 espèces culicidiennes ont été identifiées, auxquelles s'ajoute une sous-espèce endémique de l'île, $C x$. comorensis kartalae (Brunhes, 1978). Boisier et al. (1994) ont capturé sur homme en journée Ae. bromeliae, espèce relativement abondante, et trois femelles d'An. pharoensis, soit la toute première mention de cet anophèle dans l'archipel des Comores. Chauvet y signale en 1967 la présence de $C x$. andersoni, une espèce proche de $C x$. pipiens, collecté au stade larvaire. Ces deux dernières espèces d'origine afrotropicale n'ont jamais été observées sur aucune autre île de l'océan Indien et leur présence doit être confirmée par de nouvelles captures. Aedes aegypti y est abondant mais la présence d'Ae. albopictus n'est pas rapportée (Sang et al. 2008). En revanche, la présence d'une espèce endémique d'Uranotaenia du sous-genre Pseudoficalbia (Ur. comorensis, capturée en 1969 à l'état larvaire dans divers phytotelmes à différents étages d'altitudinaux) est assez remarquable compte-tenu du très faible âge géologique de Grande Comore. Cette espèce est également présente à Mayotte.

Mohéli. C'est la plus petite des quatre îles $\left(290 \mathrm{~km}^{2}\right)$. Elle culmine à $790 \mathrm{~m}$ d'altitude et est formée d'un plateau bordé de plaines côtières avec des sols argileux permettant la présence de petits cours d'eau permanents et d'eau stagnante de surface. La forêt qui couvrait près de $25 \%$ de l'île en 1955 se cantonne désormais au-delà de $500 \mathrm{~m}$ d'altitude. Toutefois, Mohéli demeure la mieux préservée de l'archipel sur le plan environnemental, à mettre en relation avec une densité de population bien moindre (99 habitants $/ \mathrm{km}^{2}$ ) que sur les autres îles. Mohéli a fait l'objet de plusieurs prospections entomologiques, malheureusement assez sommaires (Brygoo \& Escolivet 1955; Grjebine 1956a; Brunhes 1977a, 1977b ; Robert 2003 ; Marsden et al. 2013) ce qui expliquerait que seules 28 espèces sont connues de cette île a priori favorable au développement de la faune culicidienne. Sept espèces d'anophèles y sont rapportées dont Anopheles funestus, vecteur majeur de l'agent du paludisme humain. Brygoo \& Escolivet (1955) y citent, à tort, An. pauliani et An. marshallii, résultante fort probable d'une confusion avec An. maculipalpis pour la première et avec An. mascarensis pour la seconde (Grjebine 1966). Signalons également la présence de Cx. univittatus (Robert, 2003), espèce observée à Madagascar et à l'île Maurice. Mohéli est la seule île du SOOI où un mâle de $C x$. chauveti, espèce d'origine malgache, a été capturé en 1970 au niveau du village de Ouallah-Miréréni (collection ARIM, Brunhes réc.). Hormis ces deux espèces, les 26 autres sont partagées avec la faune culicidienne de Mayotte.

Anjouan. Cette île de $424 \mathrm{~km}^{2}$ présente des plaines côtières marécageuses et un relief vigoureux qui culmine à $1595 \mathrm{~m}$ (mont Ntringui). L'île possède de nombreux petits cours d'eau temporaires ou permanents et des collections d'eau stagnante ; elle héberge 332000 habitants et est la plus densément peuplée de l'archipel (517 habitants $/ \mathrm{km}^{2}$ ). Sous cette pression démographique croissante, l'île subit un défrichage important. L'érosion concomitante des sols qui en résulte se traduit par une raréfaction de ses ressources en eaux superficielles ; des 49 cours d'eau permanents recensés en 1950, il n'en demeurait qu'une trentaine en 1970 et seule une dizaine subsiste actuellement (Charmoille 2013). Brunhes (1978) notait qu'Anjouan était l'île la moins bien prospectée de l'archipel du point de vue de la faune culicidienne, et cela reste vrai de nos jours. Seules 23 espèces culicidiennes (6 Anophelinae et 17 Culicinae) y sont signalées. Ainsi, à Anjouan, comme à Mohéli, l'absence d'Uranotaenia relève 
probablement d'un déficit de prospection puisque 6 espèces appartenant à ce genre sont présentes à Mayotte (da Cunha Ramos \& Brunhes 2004 ; Le Goff et al. 2014). Culex sunyaniensis y a été récemment découvert à une embouchure de rivière polluée à Assimpao, sur la base d'une seule larve récoltée et identifiée "Cx. sunyaniensis-like" (Marsden et al. 2013), ce qui suggère une certaine incertitude dans l'identification. Cette espèce rare a aussi été mentionnée de l'île de Praslin aux Seychelles où elle a été capturée à l'état adulte et identifiée après dissection des genitalia mâles et observation de la répartition des soies du lobe subapical (Le Goff et al. 2012). Elle n'a pas été détectée dans les autres îles de l'archipel des Comores. Pareillement, Ae. cumminsii capturé au stade larvaire (Chauvet 1967), stade où cette espèce est morphologiquement proche d'Ae. fowleri, n'a jamais été retrouvé par ailleurs, tant aux Comores que sur l'ensemble de la zone d'étude. La présence d'adultes d'Ae. albopictus, vecteur majeur d'arbovirus, a été récemment rapportée pour la première fois (Marsden et al. 2013).

Mayotte. C'est la plus ancienne des îles de l'archipel des Comores (7,7 Ma). Son relief est modéré avec un point culminant à $660 \mathrm{~m}$, soit le plus faible de l'archipel. Elle présente des conditions géologiques et climatiques analogues à celles de Mohéli et Anjouan, avec un profond sol argileux qui permet la formation de mares et de quelques cours d'eau permanents. Pendant la saison des pluies, l'eau de surface est abondante.

Brygoo \& Escolivet (1955), Grjebine (1956b) et Paulian (1964) ont effectué les premiers inventaires de la faune culicidienne avec un bilan de 14 espèces dénombrées. Lors d'une étude sur la transmission de la filariose de Bancroft, Brunhes (1978) a observé 16 nouvelles espèces. Plus récemment, Le Goff et al. (2014) ont récolté 40 espèces avec 8 espèces supplémentaires dont une nouvelle pour la Science et considérée à l'heure actuelle comme endémique de Mayotte (Aedes pia). Ce sont donc au total 45 espèces qui ont été signalées sur cette île. Les espèces agressives pour l'homme de jour sont Ae. aegypti, Ae. albopictus, Er. subsimplicipes, Er. quinquevittatus et Ae. cartroni. Durant la nuit, ce sont $C x$. quinquefasciatus, An. gambiae s.s. et An. funestus qui prennent le relais. La présence d'Ae. albopictus a été détectée pour la première fois en novembre 2001 (Girod 2004), notamment sur les deux plus grandes îles habitées : Grande-Terre et Petite-Terre. Depuis, il est probable que l'espèce se soit établie sur tout l'archipel des Comores, puisque présente à Anjouan, mais cela reste à démontrer. Rappelons que $A e$. albopictus n'avait pas été capturé en 1993 sur les quatre îles, lors de l'épidémie de dengue qui frappa alors l'archipel, le vecteur incriminé étant exclusivement Ae. aegypti (Boisier et al. 1994). Aedes albopictus, avec Ae. aegypti, An. gambiae et Er. subsimplicipes sont actuellement les espèces les plus abondantes à Mayotte. La sous-espèce formosus semble être la seule sous-espèce d'Ae. aegypti présente sur l'île. Culex tritaeniorhynchus a été capturé pour la première fois sur l'île en 2013 (Le Goff et al. 2013). Culex simpsoni est présent, conformément à sa vaste répartition sur le continent africain et dans la plupart des îles du SOOI, en lien avec une large plasticité écologique acceptant des gîtes larvaires très diversifiés, naturels ou artificiels, de tailles très restreintes à vastes, dans les eaux riches en matières organiques ou salées, avec ou sans végétation. Les femelles ne piquent toutefois que rarement l'homme et ne transmettent aucun pathogène d'affection humaine. Espèce très agressive en milieu ombragée, Aedes bromeliae est assez fréquent sur l'île et majoritairement dans les habitats naturels des aisselles foliaires, en particulier Typhonodorum sp. (Araceae). Elle est un vecteur important de fièvre jaune en Afrique de l'Est. Aedes pia est une espèce présente sur l'ensemble de Mayotte. Son stade larvaire est morphologiquement proche d'Ae. aegypti, mais le stade adulte est facilement identifiable. La biologie des adultes, notamment leur rôle vecteur, reste inconnue (Le Goff et al. 2013) mais son anthropophilie de piqûre a été observée en fin de journée, entre 16 h et $19 \mathrm{~h}$ (Achirafi 2013).

Dans notre zone d'étude, $C x$. carleti, d'origine malgache, a été capturé à plusieurs reprises et exclusivement à Mayotte. Ses stades aquatiques se développant dans les phytotelmes comme les bambous fraichement coupés (Le Goff et al. 2014), cette espèce pourrait à l'avenir être rencontrée à Mohéli dans ce type de gîtes bien qu'elle n'ait jusqu'à présent jamais été trouvée tant sur Grande Comore que sur Anjouan où elle a été spécifiquement recherchée (Brunhes 1978).

Sur les neuf espèces anophéliennes peuplant les Comores, toutes sont présentes à Mayotte à l'exception d'An. pharoensis. Les espèces les plus abondantes sont Anopheles gambiae et An. pretoriensis, suivies à un niveau nettement moindre par An. funestus qui a subi une forte raréfaction de ses populations à la fin du $20^{\mathrm{e}}$ siècle (Blanchy et al. 1999 ; Elissa \& Karsh 2005). Dotée d'une forte agressivité nocturne envers l'homme, An. funestus est désormais considérée dans certaines zones de l'île comme le vecteur principal de Plasmodium humain (Maillard et al. 2015). Anopheles funestus occupe pratiquement les 
mêmes types de gîtes que ceux d'An. mascarensis (plan d'eau permanent ou semi-permanent ombragé à végétation dressée des plaines littorales) ce qui explique leur absence de Grande Comore. Cette dernière espèce est exophile et les femelles se nourrissent tant sur bétail que sur homme. Anopheles gambiae est commune à Mayotte comme sur l'ensemble des îles dans les collections d'eau souvent temporaires mais bien ensoleillées. Anopheles merus, espèce côtière d'eau saumâtre, est sporadiquement présente (2\% des captures) sur les quatre îles comoriennes (Marsden et al. 2013 ; Le Goff et al. 2014). Enfin An. pretoriensis, bien que très commune, est une espèce largement zoophile et exophage qui n'intervient pas dans la transmission du paludisme humain.

\section{Les îles Éparses}

Le terme "îles Éparses de l'océan Indien" regroupe un ensemble de cinq entités dispersées autour de Madagascar : l'île Europa, Bassas da India, l'île Juan de Nova, les îles Glorieuses (composées de l'île Grande Glorieuse et de l'île du Lys), et enfin l'île de Tromelin, cette dernière étant la seule située hors du canal du Mozambique, à $450 \mathrm{~km}$ à l'est de Madagascar. La superficie des îles Éparses est très réduite $\left(43,2 \mathrm{~km}^{2}\right.$ en surface cumulée) pour une altitude maximale de $15 \mathrm{~m}$. Ces îles sont maintenant protégées depuis leur classement en "réserve naturelle intégrale" en 1975. Europa, Bassas da India, Tromelin et l'île du Lys furent peu anthropisées, à l'opposé de Juan de Nova et de Grande Glorieuse qui ont connu au début du $20^{\mathrm{e}}$ siècle l'exploitation d'une mine de phosphate (guano) pour la première, stoppée en 1970, et une déforestation pour l'implantation d'une cocoteraie pour la seconde. Elles accueillent $40 \%$ des oiseaux marins des îles de l'océan Indien dont quelques sous-espèces endémiques. Les lézards y sont présents en grand nombre, dont des formes endémiques ainsi que d'importants cheptels de tortues vertes (Chelonia mydas). Les seuls mammifères présents résultent d'introductions par l'homme et se limitent actuellement à des chèvres redevenues sauvages, rats (Rattus norvegicus, $R$. rattus), souris (Mus musculus) et chats (Russell \& le Corre 2009). Une campagne d'éradication des chats est menée depuis 2015 à Juan de Nova (Sanchez 2015). Une mangrove de 700 ha est présente sur le lagon de l'île Europa. Comme les îles coralliennes des Seychelles, les îles Éparses furent submergées lors des périodes interglaciaires, impliquant que les faunes et flores actuelles sont de colonisation relativement récente.

Bassas da India est un atoll corallien en formation dans le canal du Mozambique ; il est constitué par un récif madréporique de moins d' $1 \mathrm{~km}^{2}$ presque totalement submergé à marée haute car culminant à 2,4 mètres d'altitude (seule une petite parcelle de terre émerge en permanence, ce qui permet de la qualifier d'île), si bien que l'atoll présente une faune et une flore strictement marines.

Douze espèces de moustiques y furent signalées depuis les années 1950 (Paulian 1950 ; Viette 1966 ; Denys 1985 ; Girod \& Le Goff 2005 ; Bagny et al. 2009b) mais seules neuf d'entre-elles furent observées ces dernières années (Tableau 3). Trois ont continuellement été présentes sur Europa et Juan de Nova : Ae. aegypti, Ae. fryeri et $C x$. sitiens. Aedes aegypti est la seule espèce présente sur les trois îles principales (Europa, Juan de Nova et Grande Glorieuse). Six espèces furent détectées ces 15 dernières années : Lutzia tigripes, Cx. quinquefasciatus, Cx. tritaeniorhynchus, Mansonia uniformis, An. gambiae s. l. et Ae. albopictus. Cette dernière a été capturée pour la première fois en 2007 à Grande Glorieuse et en 2008 à Juan de Nova dans des gîtes anthropiques (Bagny et al. 2009b). Mansonia uniformis a été signalé à Juan de Nova et An. arabiensis à Grande Glorieuse mais en faible nombre, sans doute introduit par avion depuis Madagascar ou la Réunion (Girod \& Le Goff 2005). Enfin, trois espèces ont été mentionnées pour la dernière fois dans les années 1960: Eretmapodites plioleucus mentionné uniquement de l'île Europa (Poisson 1923), Ae. fowleri et Ae. dufouri. Ces disparitions sont tout à fait énigmatiques d'autant que les deux premières (Er. plioleucus et Ae. fowleri) ont été signalées en abondance et agressives pour l'homme. Eretmapodites plioleucus se développait dans les plantations de sisal (Agave sisalana) mises en place au nord de l'île Europa vers 1905, puis abandonnées dans les années 60 mais qui couvrent encore 95 ha. Un programme d'éradication de cette plante invasive est en cours. La présence éphémère au début du $20^{\mathrm{e}}$ siècle de cette espèce afrotropicale restera incertaine puisqu'aucun spécimen n'a été conservé. La confirmation d'une diagnose correcte pour cette espèce facilement reconnaissable semble donc aujourd'hui impossible. Au final, on hésite donc pour Er. plioleucus entre une erreur de détermination et, plus probablement, une extinction locale pour une cause inconnue. Par contre, il semble bien que Ae. fowleri et Ae. dufouri furent confondus avec Ae. fryeri (Girod \& Le Goff 2005), toujours extrêmement abondant et agressif à Europa et Juan de Nova. En effet, les femelles de ces trois espèces sont morphologiquement proches, tout comme les larves d'Ae. fryeri et d'Ae. dufouri. 
Tromelin n'a pas été prospectée de façon approfondie mais Salvan en 1994 y signale la présence d'Ae. fowleri (in Julvez \& Mouchet 1994); cependant nous pensons que cette espèce a été confondue avec Ae. fryeri, comme dans les autres îles Éparses.

Aucune espèce de moustique n'est endémique des îles Éparses. Toutes sont présentes tant sur la côte Est africaine qu'à Madagascar.

\section{Les Mascareignes}

Les Mascareignes regroupent trois îles principales, toutes d'origine volcanique, la Réunion, l'île Maurice et Rodrigues. Formées entre 3 et $9 \mathrm{Ma}$, la Réunion et Maurice sont les deux îles les plus étendues de l'océan Indien. Elles sont situées par $20^{\circ}$ de latitude sud, à $700 \mathrm{~km}$ à l'est de Madagascar et à $4000 \mathrm{~km}$ de l'Inde. la Réunion est nettement la plus récente des deux îles avec un volcanisme encore actif. Son relief est extrêmement vigoureux, elle culmine à $3070 \mathrm{~m}$ et seules $25 \%$ de ses terres se situent à une altitude inférieure à $500 \mathrm{~m}$ pour une superficie de $2512 \mathrm{~km}^{2}$. Les sols perméables, de fortes pentes et des bassins versants réduits restreignent les eaux de surface à quelques rivières permanentes, à de rares mares d'altitudes et à quelques étangs littoraux. L'île Maurice est située à $180 \mathrm{~km}$ au nord-est de la Réunion. Plus petite que sa voisine, sa taille est de l'ordre de $60 \mathrm{~km}$ sur $40 \mathrm{~km}$ soit $1865 \mathrm{~km}^{2}$. Les dernières manifestations volcaniques remonteraient de 25000 à 100000 ans (Willaine 1984). Le point culminant de l'île est à $828 \mathrm{~m}$, traduisant un relief érodé : $97 \%$ des terres se situent au-dessous de 500 $\mathrm{m}$ d'altitude, donnant un paysage de plaines littorales et de plateaux où domine la culture de la canne à sucre.

Des trois îles principales de l'archipel des Mascareignes, l'île Rodrigues est la plus petite $\left(110 \mathrm{~km}^{2}\right)$. Située à $560 \mathrm{~km}$ à l'est de Maurice, elle est la plus septentrionale et la plus isolée. L'île serait, au moins pour sa partie émergée, la plus âgée des trois îles avec plus de 10 million d'années d'existence. Bien que d'élévation modeste (le plus haut sommet culmine à 398 mètres), l'île présente une topographie générale de type montagneux. Les fonds des vallées demeurent le plus souvent à sec et ne sont parcourus par des flots torrentiels que lors des fortes pluies de régime cyclonique. Sa population était de 42400 habitants fin 2016.

Île de la Réunion. Malgré les minutieuses prospections entomologiques effectuées (Hamon 1953 ; Girod 2001 ; Boussès et al. 2013), la faune culicidienne de la Réunion se limite à 12 espèces et apparait comme la plus pauvre des grandes îles de l'océan Indien (Tableau 4). Ce peuplement comprend seulement deux espèces endémiques : Or. reunionensis qui est morphologiquement très proche d'Or. arboricollis, endémique de Maurice, et Ae. dufouri, quant à lui proche d'Ae. fryeri et dont les larves sont également halophiles. Les dix autres espèces sont communes à Madagascar et au continent africain. Huit d'entre elles sont susceptibles de piquer l'homme. Certaines ont une répartition pantropicale voire cosmopolite (Ae. aegypti, Ae. albopictus, Cx. quinquefasciatus et, dans une moindre mesure, $C x$. tritaeniorhynchus), d'autres sont implantées sur le continent africain et à Madagascar (An. coustani, An. arabiensis). Tout comme dans de nombreuses îles de l'océan Indien, les espèces dominantes à la Réunion sont Ae. albopictus et $C x$. quinquefasciatus. Dans une moindre mesure, $C x$. neavei est également abondant ; très peu anthropophile, il est principalement récolté à l'état larvaire (Le Goff, observations non publiées). La population d'Ae. albopictus en place à la Réunion est génétiquement proche, entre-autres, des populations contemporaines malgaches, américaines, chinoises, japonaises ou de France métropolitaine (Mousson et al. 2005 ; Maynard et al. 2017), ce qui suggère un flux migratoire récent lié à la globalisation des échanges et à des ré-invasions multiples (Manni et al. 2017). La population actuelle a probablement remplacé la population présente au début du $20^{\mathrm{e}}$ siècle et a soumis à une forte compétition la population en place d'Ae. aegypti (Delatte et al. 2011). En effet, une particularité de la Réunion réside dans les faibles densités observées d'Ae. aegypti qui se limite désormais à quelques refuges dans des ravines sèches de la côte sous le vent et à une altitude inférieure à $500 \mathrm{~m}$. Sa raréfaction découle de la compétition avec Ae. albopictus qui le repousse vers des lieux moins anthropisés, notamment dans le fond de certaines ravines (Salvan \& Mouchet 1994). Aedes albopictus est opportuniste pour ses préférences trophiques à la fois anthropophile et zoophile. Il s'est adapté aux environnements urbains et périurbains et est extrêmement abondant sur tout le littoral habité de l'île et jusqu'à 500/700 m d'altitude, atteignant parfois $1200 \mathrm{~m}$. Il est également présent dans les périmètres agricoles, comme les champs de canne (Le Goff et al. 2016), mais également dans les ravines ombragées et espaces forestiers non habités. 
Anopheles arabiensis est la seule espèce du complexe An. gambiae présente sur l'île (Denys \& Isautier 1991 ; Girod et al. 1999 ; Boussès et al. 2013). Cette espèce fut vraisemblablement introduite sur l'île avec la mise en place en 1864 d'une ligne de navires à vapeur entre Madagascar, l'Afrique et les Mascareignes : la forte réduction de la durée des voyages entre les îles lui a permis de survivre durant les traversées (Julvez \& Mouchet 1994). Un paludisme autochtone s'est alors déployé dès 1869 à la Réunion et fut seulement éliminé dans les années 1970. Les populations d'An. arabiensis se cantonnent désormais à une altitude inférieure à $400 \mathrm{~m}$ (Gouagna et al. 2011), ayant un comportement exophile.

Malgré ses grandes dimensions et les nombreux gîtes potentiels qu'elle recèle pour les stades aquatiques, l'île de la Réunion possède une faune culicidienne relativement pauvre. Au moins trois groupes de facteurs peuvent rendre compte de cette situation. En premier lieu, l'isolement de la Réunion, située loin des masses continentales. Par ailleurs, les courants marins et les cyclones, fréquents dans la région, circulent d'est en ouest et ne rencontrent, ni ne "brossent" aucune terre pouvant être la source de nouveaux arrivants. Le deuxième groupe de facteurs relève de l'histoire de l'île. Découverte au $16^{\mathrm{e}}$ siècle, la Réunion, contrairement aux îles comoriennes, n'était pas sur une route maritime fréquentée, en particulier par le cabotage. Sur ses côtes peu hospitalières, les mouillages sont rares et souvent périlleux. De telles conditions n'ont pas favorisé les échanges maritimes et rendent peut-être compte de l'absence d'Uranotaenia, d'Eretmapodites, de Skusea, à la bioécologie larvaire particulière, et autres Culicidae qui voyagent facilement avec l'homme ou ses marchandises. Enfin, le troisième groupe de facteurs concerne son relief élevé et abrupt qui restreint considérablement les environnements favorables aux Culicidae par la baisse rapide des températures avec l'altitude.

Il est intéressant de pointer que les deux espèces endémiques, Or. reunionensis et Ae. dufouri, ne sont pas particulièrement anthropophiles. Cette observation est compatible avec l'hypothèse que leurs ancêtres sont probablement arrivés bien avant le premier peuplement humain.

Île Maurice. Les dernières prospections publiées pour cette île remontent à près de 70 ans. Ce furent celles menées par Halcrow dans les années 1950 (Halcrow 1954a), qui a comptabilisé 18 espèces, auxquelles cet auteur ajouta dans un bref article rédigé le 17 mai de cette même année $C x$. simpsoni (Halcrow 1954b), espèce sans doute découverte alors que le premier article était sous presse. Il citait pour mémoire deux autres espèces, Ae. aegypti et An. funestus (citée par Mac Gregor 1923), déjà éliminées de l'île par les traitements insecticides antipaludiques effectués à la fin des années 1940. La présence de $C x$. pipiens était par ailleurs douteuse et faisait plus vraisemblablement référence à une autre espèce du complexe Pipiens : $C x$. quinquefasciatus. Le dernier inventaire repose sur une synthèse bibliographique sans prospection sur le terrain (Mamet \& Webb-Gebert 1980), comptabilisant 17 espèces sur l'île, en omettant $C x$. simpsoni que nous retiendrons pour notre part. Soit un total de 18 espèces dont 4 endémiques : Ae. mascarensis proche d'Ae. aegypti, Ae. vinsoni proche d'Ae. albopictus, Aedes tonkingi et $O r$. arboricollis, proche d'Or. reunionensis. Les autres espèces sont à large répartition et d'origine essentiellement afrotropicale (Ae. fowleri, Lt. tigripes, Cx. univittatus, Cx. insignis) avec parfois une répartition pantropicale ( $C x$. quinquefasciatus, $C x$. tritaeniorhynchus). Quatre anophèles sont présents à Maurice : An. arabiensis est le plus abondant dans toute l'île ; An. merus affectionne les eaux saumâtres des régions côtières ; An. coustani, espèce essentiellement zoophile, peut être abondant localement en particulier près des mares et étangs permanents à végétation dressée ; An. maculipalpis reste rare (Gopaul 1995). Espèce essentiellement zoophile, ses gites larvaires de prédilection sont les eaux à végétation dressée des zones côtières. Malgré la présence d'espèces anophéliennes potentiellement vectrices, aucun cas de paludisme autochtone n'est survenu depuis 1997 (WHO 2012). Aedes albopictus est aujourd'hui abondant dans toute l'île. Il a été rapporté comme très commun dès 1908 (d'Emmerez de Charmoy 1908) mais il est possiblement présent depuis bien plus longtemps, des "moucherons noirs piquetés de blanc" ayant été signalés dès 1768 (Bernardin de Saint Pierre 1773). Il est le vecteur majeur de l'épidémie de chikungunya survenue en 2005-2006 et très probablement de l'épidémie de dengue (DENV-2) de 2009. La dynamique de cette épidémie de dengue a fait suspecter la réintroduction d'Ae. aegypti (Issack et al. 2010) mais aucune observation ultérieure de cette dernière espèce, pourtant relativement facile à identifier, n'est venue conforter cette hypothèse qui peut maintenant être écartée. Deux Culex d'origine afrotropicale sont mentionnés parmi les captures à Maurice sans être signalés des autres îles volcaniques : $C x$. poicilipes et $C x$. thalassius. Culex poicilipes a été capturé à de rares occasions sur les régions côtières de l'île Maurice. Cette espèce, qui peut piquer l'homme, est relativement commune sur le continent africain ; elle aurait disparu de l'île de la Réunion 
dans les années 70. Culex thalassius est une espèce tolérante pour une salinité atteignant $50 \%$ de celle de l'eau de mer (Rogo et al. 1985). Cette espèce majoritairement zoophile (Mamet \& Webb-Gebert 1980) est essentiellement présente dans les marais d'eau saumâtre des plaines côtières de l'île (Halcrow 1954a).

Île Rodrigues. Mac Gregor (1923), puis Halcrow (1954a) recensent sur cette île, dont on a déjà mentionné l'éloignement, les trois mêmes espèces anthropophiles : Ae. aegypti, Cx. quinquefasciatus et $C x$. simpsoni. Cette dernière était à l'époque la plus commune sur l'île, alors que Ae. aegypti est encore largement répandue (Halcrow 1954a). Anophèles et paludisme autochtone y sont absents (Mac Gregor 1923 ; Kirk \& André 1933 ; Halcrow 1954b). Encore signalé en 1980 (Mamet \& Webb-Gebert 1980), Ae. aegypti n'est plus observé à partir de 2008 (Bagny et al. 2009a). À l'inverse, Ae. albopictus est mentionné pour la première fois à Rodrigues en 1980 (Mamet \& Webb-Gebert 1980) pour être identifiée comme l'espèce dominante en 2007 (Delatte et al. 2008). Comme à la Réunion au cours du $20^{\mathrm{e}}$ siècle, Ae. aegypti semble avoir été remplacé par Ae. albopictus à l'île Rodrigues au cours des années 2000.

\section{Les archipels de Saint-Brandon et d'Agalega}

Ces deux archipels sablonneux rattachés administrativement à la République de Maurice sont d'origine corallienne, contrairement aux Mascareignes. L'archipel habité de Saint-Brandon est situé à $390 \mathrm{~km} \mathrm{au}$ nord-nord-est de l'île Maurice. Aedes aegypti y fut semble-t-il la première espèce observée il y a près d'un siècle (Mac Gregor 1923), complétée bien plus récemment par Ae. albopictus. Les deux espèces ont été observées simultanément en 1967 selon un rapport de 1970 du ministère de la Santé de Maurice (Julvez \& Mouchet 1994).

L'archipel habité d'Agalega est situé à $990 \mathrm{~km}$ au nord de l'île Maurice. Aedes albopictus est la seule espèce à y avoir été observée (Mamet \& Webb-Gebert 1980).

\section{Les îles granitiques des Seychelles}

Les îles granitiques de la zone considérée hébergent 17 taxa de Culicidae dont 5 considérés comme endémiques de ces îles (Tableau 5). Les 43 îles ou ilots granitiques de l'archipel sont les sommets émergés il y a 75 millions d'années d'une vaste plaque granitique de $43000 \mathrm{~km}^{2}$. Ils se situent près de l'équateur $\left(5^{\circ}\right.$ de latitude sud) et sont soumis à un climat tropical humide de type équatorial. Cet archipel d'une superficie totale de $244 \mathrm{~km}^{2}$ est très éloigné de toute terre continentale : $1400 \mathrm{~km}$ du continent africain, $1100 \mathrm{~km}$ de Madagascar et $2800 \mathrm{~km}$ du sous-continent Indien. Ces îles furent tardivement habitées, à partir de 1770 et la population globale actuelle est de 93000 habitants. L'île principale, Mahé, regroupe à elle seule les deux tiers des terres de l'archipel et abrite $90 \%$ de la population. Les trois autres îles majeures sont Praslin, Silhouette et La Digue. Leur relief peut être vigoureux ; Mahé culmine en effet à $930 \mathrm{~m}$. Au-dessus de $500 \mathrm{~m}$ d'altitude les sommets sont couverts de forêt et les cours d'eau permanents sont nombreux. Le sol argileux retient de nombreuses petites collections d'eau pendant la saison des pluies. Dans de telles conditions, les gîtes larvaires potentiels pour les moustiques sont nombreux et variés.

Aedes aegypti a été la toute première espèce découverte dès 1903 aux Seychelles granitiques. Theobald (1912) a signalé la présence de plusieurs espèces incluant des endémiques (ou que l'on croyait telles) : Culex scottii, Uranotaenia pandani, Ur. nepenthes. Au cours de la seconde partie du $20^{\mathrm{e}}$ siècle, les découvertes d'espèces nouvelles n'ont pas cessé. Suite aux prospections de Harper (1947), van Someren (1947) signale ainsi Culex stellatus. Mattingly \& Brown (1955) ajoutent deux taxa nouveaux pour la Science (Aedes vigilax vansomerenae, Ur. browni). Lambrecht \& van Someren (1971) récoltent pour la première fois $C x$. wigglesworthi et une nouvelle espèce, Ae. lambrechti décrite par van Someren (1971), qui se développe dans les trous de crabe comme toutes les espèces appartenant au sous-genre Skusea. La présence de $C x$. wigglesworthi nous semble douteuse car cette espèce a été collectée à un seul exemplaire en 1968 au stade larvaire à Praslin et est facilement confondue à ce stade avec l'espèce $C x$. sunyaniensis, également présente sur cette île. Yersin et al. (1999) puis Gerlach (2009) recensent 15 espèces incluant les prospections de Bin (1995) qui mentionnent pour la première fois la présence de $C x$. fuscocephala et $C x$. tritaeniorhynchus. Le Goff et al. (2012) rapportent une nouvelle prospection entomologique, portant à 17 le nombre d'espèces incluant un nouveau Culex capturé à Praslin : $C x$. sunyianensis. Dans la petite île Aride, située $10 \mathrm{~km}$ au nord de Praslin, Ae. albopictus et Cx. simpsoni ont été observés. 
Les espèces actuellement dominantes sur les îles granitiques des Seychelles sont Ae. albopictus et $C x$. quinquefasciatus (Yersin et al. 1999 ; Le Goff et al. 2012), toutes deux découvertes dès le début du $20^{e}$ siècle (Theobald 1912). À l'amorce des années 1970, tout au moins, Ae. aegypti subsistait en faible nombre dans quelques rares zones de Mahé (Lambrecht et al. 1970 ; Metselaar et al. 1980). L'espèce est devenue extrêmement rare dans les années 1990 (Bin et al. 1996). Elle n'a pas été retrouvée lors des dernières prospections menées par Vincent Robert en 2008 (Le Goff et al. 2012), si bien que se pose la question de l'élimination potentielle d'Ae. aegypti sur ces îles granitiques.

\section{Les îles coralliennes des Seychelles}

Il existe aux Seychelles une centaine d'îles coralliennes disséminées entre 230 et $1150 \mathrm{~km}$ au sud-ouest des îles granitiques. La faible élévation de ces îles associée aux variations naturelles du niveau des océans les ont soumis à des périodes de submersion. Lorsque la submersion est totale, ce qui fut le cas il y a seulement 125000 ans (Siddall et al. 2003) pour toutes les îles coralliennes des Seychelles, les conséquences en ont été évidemment l'extinction des populations terrestres, incluant les populations de moustiques. Aujourd'hui, privées d'eau douce autre que celle des précipitations, ces îles demeurent largement inhabitées par l'homme, au moins de façon permanente. Elles se répartissent en cinq ensembles, du nord au sud : les îles Amirantes, le groupe corallien méridional, le groupe Alphonse, le groupe Farquhar et le groupe d'Aldabra. Ce dernier, composé d'Aldabra, Assomption et Cosmoledo, est le plus proche du continent africain et situé $380 \mathrm{~km}$ au nord-est de l'archipel des Comores. L'atoll d'Aldabra est le plus étendu et il est déclaré patrimoine mondial par l'UNESCO depuis 1982. Les mammifères terrestres autochtones y sont strictement limités à cinq espèces de chauves-souris (Hutson 2004 ; Goodman 2008). D'autres mammifères cosmopolites y ont été introduits : les rats (Rattus rattus) et les chats (Felis silvestris) (Harper et al. 2015 ; Seabrook 1990). Les chèvres sauvages (Capra hircus), introduites à la fin du $19^{\mathrm{e}}$ siècle, ont été éradiquées de l'atoll en 2012 (Bunbury et al. 2013). La faune dominante est constituée par des milliers de tortues terrestres (Aldabrachelys gigantea gigantea) et marines (C. mydas).

Un fait marquant concernant la faune culicidienne des Seychelles, coralliennes comme granitiques, est qu'elle est actuellement dépourvue d'anophèles (Robert et al. 2011). Un total de dix espèces a été observé (tableau 6).

Le groupe d'Aldabra fut prospecté dernièrement pour ses moustiques (Le Goff et al. 2012) avec un total de huit espèces dont Aedes sp. A (au sens de Le Goff et al. 2012) qui est endémique et présent en abondance sur l'atoll d'Aldabra et l'île d'Assomption. La plupart des espèces sont d'origine africaine (Ae. albocephalus, Ae. fryeri, Cx. antennatus, Cx. simpsoni) ou malgache (Cx. scottii). Culex sitiens, espèce d'origine asiatique largement implantée en Afrique et à Madagascar, et Ae. aegypti, espèce anthropophile à répartition pantropicale, y ont été récoltées dès 1907. Toutes les espèces de la zone sont présentes à Aldabra à l'exception notable d'Ae. albopictus et de Cx. quinquefasciatus. Aedes aegypti y est par contre abondant, piquant indistinctement hommes et tortues au même titre que Ae. albocephalus et Ae. fryeri (Le Goff et al. 2012). D'autres espèces ont été signalées à Aldabra mais jamais retrouvées ; c'est le cas d'une espèce du complexe Anopheles gambiae à l'origine d'une épidémie de paludisme qui survint de juin 1930 à janvier 1931 sur les îles Assomption et Aldabra (Hermitte 1931). La présence éphémère de cette espèce, se maintenant difficilement dans ces milieux arides durant la longue saison sèche, résulte vraisemblablement d'introductions sporadiques par bateaux (Robert et al. 2011).

Peu d'informations sont rapportées de la plupart des autres ensembles coralliens des Seychelles, si ce n'est la présence de $C x$. quinquefasciatus sur les îles Farquhar et Providence, toutes deux du groupe Farquhar (Subra 1975), de Cx. quinquefasciatus capturé en 2006 et d'Ae. albopictus capturé en 1905 et 2006 sur l'île Desroches, et d'Ae. aegypti capturé en 1901 sur l'île d'Arros, deux îles du groupe Amirantes. Aedes albopictus a également été capturé en 1905 à Coëtivy (Theobald 1912 ; Gerlach 2009). En revanche, la mention de capture d'Ae. fryeri sur Coëtivy [in Julvez \& Mouchet (1994) citant Theobald (1912)] est erronée. Au total, seules dix espèces sont inventoriées sur l'ensemble des Seychelles coralliennes.

\section{L'archipel des Chagos}

Cet archipel regroupe une soixantaine d'îles d'une superficie cumulée de $60 \mathrm{~km}^{2}$ dans sept atolls. Il est apparu il y a $48 \mathrm{Ma}$ et est situé à $1800 \mathrm{~km}$ à l'est des Seychelles granitiques, proche de l'équateur, à midistance du continent africain et de l'Indonésie. Cet ensemble héberge une importante population 
d'oiseaux marins. L'homme a implanté des cocoteraies et a introduit les habituelles espèces invasives (notamment des rats et des chats). Diego Garcia, atoll principal de $40 \mathrm{~km}^{2}$ est totalement occupé depuis 1977 par une base militaire et constitue la seule île habitée de l'archipel. Les autres ensembles d'îles, dont Salomon et Peros Banhos, sont classées en réserves naturelles.

En 1912, Theobald identifia Ae. albopictus de ce dernier atoll. La filariose lymphatique était dans les années 1940 un problème sanitaire majeur aux Chagos, avec $C x$. quinquefasciatus comme vecteur (Lavoipierre 1951). Un inventaire mené en 1969 rapporta trois espèces culicidiennes (Tableau 6), toutes anthropophiles : Cx. quinquefasciatus, Ae. albopictus et Ae. aegypti (Lambrecht \& van Someren 1971). Ces trois espèces sont présentes à Diego Garcia, Salomon et Peros Banhos à l'exception d'Ae. aegypti qui n'a pas été détecté à Peros Banhos. Culex quinquefasciatus était alors porteur $(2,5 \%$ des 120 femelles examinées) de filaires non identifiées (Lambrecht \& van Someren 1971). Le dernier inventaire réalisé en 1971 s'est limité à Diego Garcia (Hutson 1981); il rapporta uniquement la présence de $C x$. quinquefasciatus et Ae. albopictus.

\section{Discussion}

Le dernier inventaire de la biodiversité des espèces de moustiques présentes dans les îles du SOOI (avec exactement la même délimitation géographique que dans la présente étude) fait par Julvez \& Mouchet (1994) totalisait 55 espèces. Notre inventaire totalise 73 espèces, soit un accroissement de $33 \%$ en un peu plus d'une vingtaine d'années. La responsabilité historique de l'homme dans l'introduction de nouveaux moustiques dans ces îles est bien documentée.

Que représentent 73 espèces ? Ceci représente $2 \%$ du nombre total des espèces valides de moustiques, 3557 selon le site de référence Mosquito-Taxononomic-Inventory (consulté le 23 octobre 2017). Mais il n'y a aucun doute que ces îles sont des hotspots de biodiversité culicidienne. La part d'espèces endémiques de la zone ( 20 espèces sur 73 , soit $27 \%$ ) apparaît considérable.

La discussion ci-après porte notamment sur l'origine des peuplements, les divers niveaux taxonomiques, les répartitions d'espèces par groupes d'îles, l'importance de l'homme comme acteur de dispersion, ainsi que sur les espèces précédemment observées qui semblent aujourd'hui éteintes.

\section{Origine exogène ou endémique des peuplements culicidiens}

La zone géographique étudiée relève de la zone biogéographique afrotropicale (Paulian 1961 ; Julvez \& Mouchet 1994). Cet inventaire des espèces de moustique dans les îles du SOOI confirme que la majorité des espèces (46 espèces, soit $63 \%$ ) présente une affinité pour l'Afrique tropicale et/ou Madagascar (Figure 2). Il est intéressant de souligner que, en contradiction avec la superficie et la diversité relatives du continent africain et du sous-continent malgache, l'apport des espèces strictement malgaches (12 espèces) est supérieur à celui des espèces strictement africaines (2 espèces). L'apport de l'Asie est plus discret, mais il s'exprime avec des espèces à large répartition en Asie-Afrique-Madagascar (3 espèces) ou plus strictement asiatique ( 1 espèce).

Plus finement, les 52 espèces occupant l'archipel des Comores sont partagées avec Madagascar pour $70 \%$ d'entre-elles et pour $51 \%$ avec le continent africain. Les 17 espèces occupant les Seychelles granitiques sont partagées avec Madagascar pour $65 \%$ d'entre-elles et pour $53 \%$ avec le continent africain. Les 14 espèces occupant les îles coralliennes (Eparses, Seychelles coralliennes et Chagos) sont partagées avec Madagascar pour $93 \%$ d'entre-elles et pour $86 \%$ avec le continent africain.

L'endémisme propre aux îles considérées dans cette étude concerne 22 taxa (21 espèces et la sous-espèce Ae. vigilax vansomerenae), soit $30 \%$. Ce pourcentage est considérable mais il n'est pas surprenant dans un contexte insulaire, par définition fragmenté et a priori propice aux mécanismes de la spéciation. À noter que cet endémisme s'applique soit à une seule île (ex. Ae. pia à Mayotte; Ae. mascarensis à Maurice) ou à plusieurs (ex. Or. comorensis dans les quatre îles de l'archipel des Comores ; Ur. pandani dans les quatre îles principales des Seychelles granitiques). Ce taux d'endémisme reste toutefois bien en deçà de celui observé à Madagascar (59 \%) (Tantely et al. 2016). Cet endémisme touche des espèces aux niches écologiques très particulières, comme les phytotelmes pour les Uranotaenia du sous-genre Pseudoficalbia ou bien les espèces du genre Orthopodomyia, ou des milieux aquatiques salés spécifiques (trou de rocher ou de corail, trou de crabe) où parviennent à se développer des espèces halophiles (Aedes sp A, Ae. dufouri, Ae. lambrechti).

\section{Biodiversité culicidienne en fonction des sous-familles et tribus}


La sous-famille des Anophelinae est totalement absente des Seychelles et largement absente des îles coralliennes. Elle est minoritaire sur l'ensemble de la zone d'étude avec dix espèces $(10 / 73=14 \%)$ dont neuf du sous-genre Cellia et une du sous-genre Anopheles (An. coustani). Mais ce pourcentage (14\%) est conforme à ce qui est observable dans le monde (485 espèces d'Anophelinae rapportées aux 3557 espèces de Culicidae, soit également $14 \%$ ). Six espèces d'anophèles sont exclusivement rencontrées sur l'archipel des Comores. Il est important de souligner qu'il n'y a pas eu de colonisation sur une longue période des espèces anophéliennes sur les îles d'origine corallienne ou granitique. Les rares exceptions concernent une colonisation récente ou sporadique, due à une introduction accidentelle par l'homme. C'est le cas d'An. gambiae s.s. à Europa ou d'An. gambiae s.l. à Aldabra et Assomption dans les années 1930. C'est aussi, probablement le cas à Grande Glorieuse.

La sous-famille des Culicinae est représentée par 63 espèces $(63 / 73=86 \%)$ pour six tribus : Aedini, Culicini, Ficalbiini, Mansoniini, Orthopodomyiini et Uranotaeniini :

- La tribu des Aedini est représentée par 22 espèces réparties en 20 Aedes et deux Eretmapodites. Trois espèces sont presque partout présentes : deux Aedes du sous-genre Stegomyia à large répartition mondiale (Ae. aegypti et Ae. albopictus) ainsi qu'une espèce bien représentée dans la région éthiopienne (Ae. albocephalus). L'archipel des Comores et les Mascareignes regroupent la quasi-totalité des taxa (18 espèces) à l'exception notable de quatre taxa dont l'écologie larvaire est intimement liée aux trous de rocher très ensoleillés (Ae. fryeri, Ae. vigilax vansomerenae et Ae. sp. A) ou bien aux trous de crabe (Ae. lambrechti). Le nombre d'espèces d'Aedini est assez modeste sur les îles coralliennes (cinq espèces) comme sur les Seychelles granitiques (cinq espèces). Certains sous-genres malgaches ou africains ne sont pas représentés sur ces îles, en particulier les Diceromyia africains et leurs proches cousins malgaches les Paulianius (Brunhes et al. 2017).

- La tribu des Culicini est représentée par 26 espèces réparties en 25 Culex et une Lutzia. Trois Culex du sous-genre Culex sont largement réparties dans les îles ( $C x$. quinquefasciatus, $C x$. simpsoni et $C x$. tritaeniorhynchus) et même au-delà puisqu'à très large répartition régionale ou mondiale. L'archipel des Comores et les Mascareignes hébergent 24 espèces de la tribu, soit beaucoup plus que dans les Seychelles granitiques ou les îles coralliennes (respectivement 8 et 7 espèces).

- La tribu des Ficalbiini est représentée par une seule espèce (My. grjebinei), présente uniquement dans deux îles de l'archipel des Comores.

- La tribu des Mansoniini est représentée par une seule espèce (Ma. uniformis), présente sur l'archipel des Comores, les Seychelles granitiques et les îles Éparses. Mais sa présence sur l'île de Juan de Nova, aux Éparses, semble sporadique, voire accidentelle.

- La tribu des Orthopodomyiimi est représentée par quatre espèces du genre Orthopodomyia, endémiques, peuplant uniquement les îles de l'archipel des Comores ou des Mascareignes.

- La tribu des Uranotaeniini est représentée par neuf espèces du genre Uranotaenia dont quatre du sousgenre Pseudoficalbia et cinq du sous-genre Uranotaenia. Six espèces (une du sous-genre Pseudoficalbia et cinq du sous-genre nominatif), toutes d'origine malgache, peuplent exclusivement des îles de l'archipel des Comores. Enfin, trois Pseudoficalbia endémiques peuplent les îles granitiques des Seychelles.

A noter que la tribu des Toxorhynchitiini, pourtant largement représentée sur le continent africain et à Madagascar est totalement absente des îles du SOOI. En particulier à Mayotte et à Anjouan pour les espèces malgaches du groupe Pauliani (sous-genre Afrorhynchus et genre Toxorhynchites) qui y auraient trouvé des gîtes convenables au développement de leurs stades préimaginaux.

\section{Biodiversité culicidienne en fonction des groupes d'îles}

L'archipel des Comores. Le nombre d'espèces culicidiennes présentes dépend largement de l'ancienneté des îles, de leur éloignement par rapport à une terre continentale et de leur position par rapport aux vents et courants dominants ainsi qu'à leur fréquentation par le cabotage. Ceci est largement vérifié dans l'archipel des Comores. La Grande Comore est une île au volcanisme encore actif et à sol poreux si bien que l'eau douce ne stagne pas en surface. Elle présente un peuplement culicidien réduit (19 espèces) malgré une position "au vent" venant de Madagascar qui est susceptible de lui apporter des espèces malgaches. Par ailleurs, un important et très ancien cabotage entre les côtes africaines et malgaches aurait pu aussi enrichir sa faune culicidienne. Il n'en a rien été car les gîtes larvaires possibles sont rares sur cette île. A l'inverse, les îles de Mayotte et de Mohéli, pourtant toutes proches et plus petites que la Grande Comore, possèdent des peuplements beaucoup plus riches, avec respectivement 
45 et 28 espèces. Par ailleurs, Mayotte est probablement en contact maritime avec Madagascar depuis plus longtemps que les autres îles de l'archipel. La parenté des populations humaines présentes sur l'île en atteste (Ottino 1974 ; Allibert 2015). Ces fréquentes arrivées de moustiques malgaches, plus rarement africains, couplés avec une île offrant de nombreux gîtes larvaires potentiels rendent compte de la richesse du peuplement mahorais. A Mayotte comme à Mohéli, un volcanisme ancien, avec une rochemère largement transformée aboutissant à un sol imperméable, permet le maintien de gîtes larvaires nombreux et variés. La comparaison entre Grande Comore et Mayotte, soumises à un flux migratoire comparable d'espèces, permet de relativiser l'importance du soubassement géologique, dans les deux cas volcanique, à l'avantage de caractéristiques pédologiques, entre une quasi absence de sol sur des laves relativement récentes à Grande Comore versus un sol profond et imperméable à Mayotte.

Dans l'archipel des Comores, on compte 53 espèces, soit près de $72 \%$ des espèces culicidiennes de la zone ; 34 d'entre-elles ( $46 \%$ du total) ne se rencontrent d'ailleurs que sur cet archipel. Elle a une affinité biogéographique plus grande avec Madagascar qu'avec le continent africain (avec en commun 36 espèces $v s 28$ espèces, respectivement). Cette forte présence d'espèces malgaches, anthropophiles ou non, est probablement la double conséquence de vents porteurs soufflant d'est en ouest et d'activités commerciales humaines (Le Goff et al. 2014). La remarquable richesse constatée de la faune culicidienne de Mayotte est certes le fait d'un effort soutenu de prospection ; mais elle est due également à l'ancienneté de l'île, à son élévation permettant la variété des milieux naturels, aux nombreux gîtes potentiels diversifiés, à la proximité de Madagascar, au très ancien commerce maritime par boutres à voile entre Madagascar et Mayotte. Cependant, cette observation n'a pas valeur de règle stricte et pour exemple nous citerons les populations d'An. funestus peuplant les Comores qui ne sont pas originaires de Madagascar mais du continent africain (Ayala et al. 2006), tout comme sans doute les populations d'An. gambiae (Petrarca et al. 1990).

Les prospections peu nombreuses et un sol maigre très perméable concourent logiquement au nombre modéré d'espèces de moustiques recensées à Grande Comore. La plupart des espèces rencontrées sur Grande Comore, Anjouan et Mohéli sont présentes à Mayotte, à l'exception de sept d'entre-elles dont la sous-espèce endémique de Grande Comore, $C x$. comorensis kartalae.

Huit espèces ou sous-espèces sont endémiques de l'archipel des Comores (Ae. brunhesi, Ae. pia, An. comorensis, Cx. comorensis kartalae, Or. comorensis, Or. joyoni, Mi. grjebinei, Ur. comorensis). Un tel niveau d'endémicité dans un si petit archipel est remarquable. Il résulte de l'isolement et de la relative ancienneté de ces îles volcaniques et tout particulièrement de Mohéli et de Mayotte. La plupart de ces espèces endémiques dérivent d'espèces malgaches. Ainsi, Mi. grjebinei est taxonomiquement et phylogéographiquement très proches de l'espèce malgache Mi. roubaudi (Brunhes 1977b). De même, les Orthopodomyia et Ur. comorensis endémiques de l'archipel des Comores relèvent, respectivement, $\mathrm{du}$ groupe Vernoni et du groupe Nigripes du sous-genre Pseudoficalbia, groupes qui sont très bien représentés dans l'ouest de Madagascar. Parfois, la proximité taxonomique des espèces est telle que les caractères morphologiques seuls sont délicats, voire insuffisants pour les identifier : tel est le cas du complexe d'espèces Ae. monetus (du sous-genre Zavortinkius) à Mayotte. Le niveau d'expertise taxonomique dont ont bénéficié les prospections entomologiques de cette île a favorablement joué sur la bonne connaissance actuelle de sa faune culicidienne.

Les îles coralliennes (îles Eparses, Seychelles coralliennes et Chagos). Les îles coralliennes offrent aux moustiques une faible variété d'habitats pour les stades aquatiques et aériens si bien qu'il n'est pas surprenant d'y rencontrer seulement des espèces halophiles et des espèces dont les œufs résistent à de longues périodes de sécheresse. Les peuplements culicidiens de ces îles sont donc pauvres, en relation directe avec l'absence d'eau douce, et occupent des niches écologiques bien spécifiques qui s'inscrivent dans l'importante diversité biologique de la zone d'étude, au même titre que leurs hôtes vertébrés (tortues, squamates, oiseaux, etc...). Il faut également souligner que les îles coralliennes habitées peuvent accueillir, pour des périodes plus ou moins longues, des espèces culicidiennes dont l'écologie larvaire est adaptée à l'eau douce et/ou des gîtes larvaires artificiels apportées par l'homme.

Les moustiques ont deux options pour s'implanter sur ces îles coralliennes. Ils doivent posséder la capacité de se développer dans l'eau saumâtre présente en permanence dans les trous de crabes et aux abords de mangroves. La seconde option concerne à la fois leurs œufs qui doivent résister à la dessiccation et la durée du développement larvaire qui doit être rapidement achevé avant l'assèchement des petits creux dans le calcaire corallien. Dans ce cas, les femelles peuvent pondre sur les bords des petites cavités rocheuses. 
Les espèces les plus fréquemment rencontrées sur ce type d'île sont Ae. aegypti et Ae. albopictus dont les œufs sont résistants à la dessiccation et tolèrent une faible dose de sel apportée par les embruns. Il n'est pas surprenant d'y rencontrer aussi des espèces dont les larves sont halophiles comme $A e$. albocephalus, Ae. fryeri, Ae. sp. A et Cx. tritaeniorhynchus. Ces espèces se développent essentiellement dans les petites cavités que l'eau de pluie, légèrement acide, creuse dans le calcaire. Pendant la saison des pluies, sur l'île Europa, les femelles agressives d'Ae. fryeri peuvent atteindre des densités spectaculaires (Girod \& Le Goff 2005). Les repas de sang des femelles sont essentiellement pris sur les oiseaux, lézards et tortues mais également sans doute sur les petits rongeurs souvent introduits sur ce type d'habitat.

Il est surprenant de constater qu'aucune des quatre espèces d'Aedes du sous-genre Skusea (Ravaonjanahary \& Brunhes 1977) qui sont présentes sur les côtes malgaches, à Mayotte ou aux Seychelles granitiques, et dont les stades aquatiques exploitent les trous de crabe, ne se soient implantées sur l'une de ces îles. Les crabes fouisseurs y sont pourtant très abondants et il arrive même qu'une mangrove soit présente.

À l'opposé, la présence de $C x$. antennatus et de Lt. tigripes sur certaines îles coralliennes est surprenante. Culex antennatus, même si elle tolère une eau légèrement salée, n'est pas une espèce halophile et ne présente pas d'œufs résistants à la dessiccation. Quant à Lt. tigripes, elle ne tolère pas les milieux salés d'une concentration supérieure à $4 \mathrm{~g} / 1$ (Ribeiro \& da Cunha Ramos 1980). Ces espèces se maintiennent peut-être en pondant dans de l'eau douce liée à la présence humaine. Elles peuvent aussi résulter d'importations récentes, multiples ou non, sans assurance de s'implanter durablement. Pareillement, la capture d'un unique spécimen de Ma. uniformis faite dans un piège lumineux à Juan de Nova est totalement inattendue sur ce type d'îlot (Girod \& Le Goff 2005). Les larves de cette espèce se développent en effet en eau douce et respirent à travers les tubes aérifères des plantes aquatiques, en se fixant par l'extrémité de leur siphon sur les racines immergées alors que de tels habitats sont rares dans ce type d'île.

Les Mascareignes. Dans les Mascareignes, malgré une forte diversité de biotopes fournissant de multiples gîtes, la grande similitude entre les peuplements culicidiens de la Réunion et de Maurice est à relier à leur proximité géographique relative. Leur diversité relativement faible (au moins par rapport aux îles de l'archipel des Comores) est à relier à leur important éloignement du continent africain et de Madagascar et à leur exposition aux vents dominants qui proviennent d'immenses étendues maritimes dépourvues de sources de Culicidae. Avant l'arrivée des premiers hommes, les trois îles principales des Mascareignes devaient être particulièrement pauvres en espèces culicidiennes.

Les îles granitiques des Seychelles. Ces îles constituent un cas particulier dans la zone géographique étudiée, étant les seules îles à substrat granitique. Elles sont à la fois anciennes, accueillantes pour une faune culicidienne variée car l'eau de surface y est abondante et le climat chaud, mais elles sont très isolées dans l'océan Indien. Ces caractéristiques peuvent rendre compte de leur peuplement culicidien réduit, dû principalement à l'isolement, que confirme la présence de cinq espèces endémiques sur les 18 espèces présentes.

Il faut souligner l'absence d'espèces anophéliennes aux Seychelles granitiques et donc l'absence de tout paludisme autochtone, la dizaine de cas annuels enregistrés étant des cas importés provenant majoritairement de travailleurs indiens expatriés (Robert et al. 2011).

Les îles granitiques des Seychelles offrent aux larves de moustiques de nombreux gîtes larvaires originaux, dont les phytotelmes. Une très ancienne implantation a ainsi permis aux trois espèces d'Uranotaenia d'évoluer dans cette gamme très diversifiée de gites et de former trois espèces endémiques : Ur. nepenthes, Ur. pandani et Ur. browni. Des espèces morphologiquement proches mais distinctes, utilisant les mêmes types de gîtes s'observent à Madagascar, aux Comores et en Asie du SudEst. Un long isolement, associé à la dérive génétique qui l'accompagne, a ainsi permis l'apparition d'espèces nouvelles. Il en est de même pour les Aedes du sous-genre Skusea.

A côté de ces espèces anciennes qui ont évolué sur place se rencontrent des espèces introduites récemment par l'homme. Il n'est en l'état pas possible de préciser si ces espèces sont arrivées à l'état adulte en utilisant les moyens de transport humains, à l'état d'œufs si l'espèce supporte la dessiccation, ou encore à l'état larvaire dans l'eau douce accompagnant les activités humaines. Il en est ainsi pour Ae. aegypti, Ae. albopictus, Cx. quinquefasciatus, Cx. fuscocephala et Cx. tritaeniorhynchus. Ces deux dernières espèces d'origine asiatique s'étant probablement implantées plus récemment que les autres aux Seychelles, dans les années 1990 (Bin 1995). 
Les espèces maintenant considérées comme éteintes ou éliminées des îles

Ces espèces peuvent être présentées en cinq groupes.

- Deux espèces endémiques rarissimes, comme il a été dit précédemment, ne sont pas encore considérées comme éteintes, An. comorensis à Mayotte et Ae. vinsoni à Maurice. Il nous semble probable que ce statut est appelé à changer dans les décennies à venir.

- L'espèce Er. plioleucus a été mentionnée en abondance à Europa dans les années 1960 mais plus jamais observée par la suite. Ce fait est en faveur d'une élimination locale. Il est intéressant de noter que c'est justement une île petite et à faible diversité d'habitats qui a été le cadre de cette disparition.

- A contrario, la disparition de $C x$. poicilipes de la Réunion ne relève peut être pas des mêmes causes, puisque l'île est relativement grande et offre des biotopes assez diversifiés. Culex poicilipes y fut découvert en faible densité en 1952 en divers points de l'île (Hamon 1953, collection ARIM), tant côtiers que d'altitude. Ses toutes dernières captures datent de 1973 et 1974 à Sainte-Suzanne, Saint-Gilles-lesBains et Sainte-Anne (Boussès et al. 2013). Culex poicilipes n'a donc jamais été retrouvé depuis 40 ans, alors qu'il est présent en Afrique de l'Est et à Madagascar et, plus discrètement, à l'île Maurice. Il n'a par contre jamais été cité de l'archipel des Comores et des autres îles de l'océan Indien. En conclusion, si $C x$. poicilipes semble bien éliminé de la Réunion, la cause de cette disparition reste inconnue.

- Mieux documentée cette fois, l'élimination volontaire d'une espèce du complexe Anopheles gambiae impliquée dans une épidémie de paludisme dans les années 1930 sur les îles Assomption et Aldabra. Ce point mérite discussions. Les succès complets d'une lutte antivectorielle aboutissant à l'élimination d'un vecteur sont si rares qu'il est permis de se demander si l'établissement récent de l'espèce (supposé avoir été réalisé un petit nombre d'années auparavant) et le caractère peu hospitalier de ces îles pour les anophèles, au moins pendant la longue saison sèche n'ont pas été d'un plus grand impact pour le succès de l'élimination que les actions de lutte antivectorielle. D'autant qu'une épidémie précédente de paludisme en 1908 avait elle aussi été suivie par l'élimination totale du ou des vecteurs qui sont restés inconnus [voir Robert et al. (2011), pour plus de détails]. C'est sans doute dans le même contexte que les adultes et larves d'An. arabiensis détectés à Grande Glorieuse (îles Eparses) en 2002 n'ont pas été retrouvé ultérieurement.

- Enfin, la réduction progressive et continuelle des densités d'Ae. aegypti au profit d'Ae. albopictus suggère que Ae. aegypti serait à divers stades de pré-élimination, en particulier aux Seychelles granitiques, sur l'île Rodrigues et à la Réunion. L'élimination d'Ae. aegypti semble certaine à Rodrigues et à Maurice. Alors qu'à la Réunion, l'élimination n'est pas totale. Il sera de plus haut intérêt de suivre cette situation à l'avenir pour observer si l'élimination d'Ae. aegypti va se poursuivre ou si, au contraire, un équilibre déjà stable entre les deux espèces y est déjà instauré. Un tel suivi serait aussi à mener dans l'archipel des Comores, où l'implantation d'Ae. albopictus est toute récente.

Ces îles constituent un domaine privilégié pour de telles observations d'évènements de colonisation et d'extinction. Mais chacun voit à quel point ces études sont dépendantes des observations de terrain. Les efforts d'échantillonnage réalisés ont été éminemment variables selon les époques et les îles. Par exemple, Mayotte a été plutôt bien inventoriée, alors que c'est plutôt le contraire pour l'île voisine d'Anjouan. Ceci est regrettable car d'intéressantes comparaisons sur les peuplements respectifs de ces deux îles sœurs seraient à mener.

\section{Les espèces invasives de la zone d'étude}

Bien que déjà présentes dans la zone d'étude, deux espèces invasives, $C x$. tritaeniorhynchus et Ae. albopictus, ont vu leur répartition grandement évoluer ces dernières décennies.

Découvertes tardivement aux Seychelles granitiques (Bin 1995) deux espèces d'origine orientale, $C x$. fuscocephala et $C x$. tritaeniorhynchus ont connu deux destins divergents. Cx. fuscocephala pourrait avoir disparu depuis, puisque non retrouvé sur cet archipel en 2008. Par contre, Cx. tritaeniorhynchus semble désormais installé sur Mahé et Praslin. Vecteur important du virus de l'encéphalite japonaise en Asie, son introduction dans cet archipel isolé découle vraisemblablement de l'intensification des échanges internationaux. Cx. tritaeniorhynchus est ainsi apparu sans doute récemment sur l'île de Mayotte (Le Goff et al. 2013) alors qu'il est toujours considéré absent des autres îles comoriennes. Pareillement, sa découverte récente sur deux îles Eparses, Juan de Nova (1985 et 2008) et Grande Glorieuse (2002), confirme son extension dans cette partie du monde. 
Découlant également de l'intensification des échanges internationaux, les populations initiales d'Ae. albopictus ont été remplacées ces dernières décennies par des populations nouvelles pourvues d'une plus grande plasticité écologique. Cette espèce invasive a poursuivi son extension dans le SOOI, colonisant l'archipel des Comores et des îles Eparses. Elle est désormais implantée sur 18 des 28 îles de la zone d'étude.

\section{Conclusion}

Cette région du monde apparaît comme particulièrement intéressante du point de vue de la biodiversité culicidienne. Cette biodiversité est importante en soi, mais aussi au regard du rôle vecteurs de nombreux moustiques pour de nombreux agents pathogènes viraux ou parasitaires, impactant aussi bien la santé humaine qu'animale.

Malgré le rôle de l'homme dans la dissémination des moustiques, la distribution des espèces entre les îles reste largement structurée. Les quelques espèces à distribution mondiale et largement invasives telles que Ae. aegypti, Ae. albopictus et $C x$. quinquefasciatus font logiquement exception.

Cet article de synthèse démontre que la connaissance de la distribution des moustiques dans le SOOI progresse et que si notre connaissance de la faune culicidienne dans cette région du monde s'est améliorée, c'est principalement le résultat d'efforts d'échantillonnage entomologiques constants et répétés sur le terrain.

\section{Remerciements}

Nous sommes très reconnaissants envers MM. Jacques Brunhes et Raheem Gopaul pour l'intérêt qu'ils portent à la taxonomie des moustiques de cette région du monde et le partage d'informations. Nous remercions également pour leur excellente collaboration les collègues entomologistes de l'océan Indien, notamment : Simon Julienne du Ministère de la Santé des Seychelles, Ambicadutt Bheecarry du Ministère de la Santé à Maurice, Jean-Sébastien Dehecq de l'ARS-OI à la Réunion, Betty Zumbo et Cyrille Ceherz de l'ARS-OI à Mayotte, Ouledi Ahmed de l'Université des Comores.

\section{Références}

Achirafi M. 2013. Etude de la bio-écologie d'Aedes n. sp., espèce de moustique endémique à Mayotte et vecteur potentiel d'arboviroses. Université de Tours. (rapport Master 1).

Allibert C. 2015. L'archipel des Comores et son histoire ancienne. Essai de mise en perspective des chroniques, de la tradition orale et des typologies de céramiques locales et d'importation, Afriques. Mis en ligne le 25 décembre 2015. consulté le 12 juillet 2017. URL: http:// afriques.revues.org/1721; doi: 10.4000/afriques.1721.

Ayala D, Le Goff G, Robert V, de Jong P, Takken W. 2006. Population structure of the malaria vector Anopheles funestus (Diptera: Culicidae) in Madagascar and Comoros. Acta Tropica. 97:292-300.

Bagny L, Delatte H, Elissa N, Quilici S, Fontenille D. 2009a. Aedes (Diptera: Culicidae) vectors of arboviruses in Mayotte (Indian Ocean): distribution area and larval habitats. Journal of Medical Entomology. 46(2):198-207.

Bagny L, Freulon M, Delatte H. 2009b. Première mention d'Aedes albopictus, vecteur d'arbovirus, dans les îles Éparses du canal du Mozambique et actualisation de l'inventaire de la faune culicidienne. Bulletin de la Société de Pathologie Exotique. 102(3):192-198.

Bernardin de Saint-Pierre JH. 1773. Voyage à l'Isle de France, à l'Isle de Bourbon, au Cap de Bonne-Espérance, etc. avec des observations nouvelles sur la nature et sur les hommes, par un officier du Roi. Ed. Neuchâtel. http://gallica.bnf.fr/ark:/12148/bpt6k 1019923.

Bin F. 1995. New mosquitoes in Seychelles. Seychelles Medical and Dental Journal. 1(4):6-7.

Bin F, Laporte T, Julienne S. 1996. Mosquito larval density survey on Mahé in 1995. Seychelles Medical and Dental Journal. 4(1):21-23.

Blanchy S, Julvez J, Mouchet J. 1999. Stratification épidémiologique du paludisme dans l'archipel des Comores. Bulletin de la Société de Pathologie Exotique. 92(3):177-184

Boisier P, Morvan JM, Laventure S, Charrier N, Martin E, Ouledi A, Roux J. 1994. Epidémie de dengue 1 sur l'île de la Grande Comore (République Fédérale Islamique des Comores) mars-mai 1993. Annales de la Société Belge de Médecine Tropicale. 74:217-229.

Boussès P, Dehecq S, Brengues C, Fontenille F. 2013. Inventaire actualisé des moustiques (Diptera : Culicidae) de l'île de la Réunion, océan Indien. Bulletin de la Société de Pathologie Exotique. 106:113-125.

Brunhes J. 1977a. Les moustiques de l'archipel des Comores : 1. Inventaire, répartition et description de quatre espèces ou sous-espèces nouvelles. Cahiers ORSTOM, Série Entomologie Médicale et Parasitologie. 15(2):131-152.

Brunhes J. 1977b. Les moustiques de l'archipel des Comores : 2. Description de quatre espèces nouvelles ou peu connues, répartition des membres du sous-genre Skusea dans l'océan Indien occidental, affinités de la faune culicidienne des Comores. Cahiers ORSTOM, Série Entomologie Médicale et Parasitologie. 15(2):153-70. 
Brunhes J. 1978. Les insectes hématophages de l'archipel des Comores (Diptera Culicidae, Ceratopogonidae, Simuliidae, Tabanidae, Hippoboscidae et Muscidae Stomoxyinae ; Hemiptera Cimicidae), maladies transmises et méthodes de lutte. Mémoires du Muséum National d'Histoire Naturelle. Série A: Zoologie. 109:193-246.

Brunhes J, Hervy JP. 1995. Insectes Diptères Culicidae Culicinae. Genre Orthopodomyia de la sous-région malgache et de la région afrotropicale. Faune de Madagascar n ${ }^{\circ} 85 ; 119 \mathrm{p}$.

Brunhes J, Le Goff G, Geoffroy B. 1997. Anophèles afro-tropicaux. I. - Descriptions d'espèces nouvelles et changements de statuts taxonomiques (Diptera : Culicidae). Annales de la Société Entomologique de France (N. S.). 33(2):173-183.

Brunhes J, Boussès P, Tantely ML, Kengne P. 2017. Un nouveau genre de Culicidae (Diptera), Paulianius n. gen., avec la description de trois nouvelles espèces malgaches, Annales de la Société Entomologique de France (N. S.). 53(5):344373.

Brygoo ER, Escolivet J. 1955. Enquête sur la filariose aux Comores à Mayotte et à Mohéli. Bulletin de la Société de Pathologie Exotique. 48(6):833-838.

Bunbury N, von Brandis R, Currie J, Jean-Baptiste M, Accouche W, Souyave J, Haupt P, Fleisher-Dogley F. 2013. Goats eradicated from Aldabra Atoll. Allien, the invasive species bulletin. 33:18-22.

Chapuis JL, Frenot Y, Lebouvier M. 2002. Une gamme d'îles de référence, un atout majeur pour l'évaluation de programmes de restauration dans l'archipel de Kerguelen. La Terre et la Vie-Revue d'Écologie. 9:121-130.

Charmoille A. 2013. Ebauche du fonctionnement hydrogéologique de l'Ile d'Anjouan (Comores) : Typologie des ressources en eau disponibles et discussion sur l'impact de la déforestation. [Rapport Technique] ONG AVSF, ONG Dahari <hal01465276>.

Chauvet G. 1967. Mission entomologique dans l'archipel des Comores (juillet-août 1967). Rapport ORSTOM, Tananarive ; $38 \mathrm{p}$.

Clements AN. 2012. The biology of mosquitoes. Vol 3 Transmission of viruses and interactions with bacteria. London School of Hygiene and Tropical Medicine ; $571 \mathrm{p}$.

da Cunha Ramos H, Brunhes J. 2004. Insecta Diptera Culicidae Uranotaenia. Collection Faune de Madagascar $n^{\circ} 91$. IRD Edition. Paris : CIRAD, Publications Scientifiques du MNHN ; 463 p.

Delatte H, Paupy C, Dehecq JS, Thiria J, Failloux AB, Fontenille D. 2008. Aedes albopictus, vecteur des virus du Chikungunya et de la Dengue à la Réunion: biologie et contrôle. Parasite. 15:3-13.

Delatte H, Bagny L, Brengue C, Bouetard A, Paupy C, Fontenille D. 2011. The invaders: phylogeography of dengue and chikungunya viruses Aedes vectors, on the South West islands of the Indian Ocean. Infection, Genetics and Evolution. 11(7):1769-81.

d'Emmerez de Charmoy 1908. On three new species of Culex collected during the anti-malarial campaign in Mauritius in 1908. Annals of Tropical Medicine and Parasitology. 2(3):257-264.

Denys JC. 1985. Rapport de mission effectuée les 31 octobre et 1er novembre 1985 à Europa et à la Réunion. DDASS ; 6 p.

Denys JC, Isautier H. 1991. Le maintien de l'éradication du paludisme dans l'île de la Réunion (1979-1990). Annales de la Société Belge de Médecine Tropicale. 71:209-19

Eaton AE. 1879. Observations on the insects collected in Kerguelen Island. Philosophical Transactions of the Royal Society of London. 168:228-229.

Elissa N, Karch S. 2005. Reemergence of Anopheles funestus and its possible effect on malaria transmission on Mayotte island, Indian Ocean. Journal of the American Mosquito Control Association. 21(4):472-473.

Evenhuis NL. 2007. Catalog of the Diptera of Australasia and Oceanian regions. Appendix I. Diptera of Antarctica and Subantarctic Islands. Bishop Museum Press; 797-804.

Gerlach J. 2009. The Diptera of the Seychelles Islands. Sofia, Bulgaria, Pensoft Publishers ; 431 p.

Girod R. 2001. La lutte contre la réintroduction du paludisme à la Réunion: étude entomoépidémiologique des facteurs de risque de reprise de la transmission autochtone : apport des systèmes d'information géographique [Thèse PhD]. Université de la Réunion, https://tel.archives-ouvertes.fr/tel-00575039/fr/.

Girod R. 2004. First record of Aedes albopictus in Mayotte island Comoros archipelago. Parasite. 11:74.

Girod R, Salvan M, Simard F, Andrianaivolambo L, Fontenille D, Laventure S. 1999. Évaluation de la capacité vectorielle d'Anopheles arabiensis (Diptera: Culicidae) à l'île de la Réunion : une approche du risque sanitaire lié au paludisme d'importation en zone d'éradication. Bulletin de la Société de Pathologie Exotique. 92(3):203-209.

Girod R, Le Goff G. 2005. Inventaire actualisé des moustiques (Diptera: Culicidae) des îlots français de Europa, Juan de Nova et Grande Glorieuse (Canal du Mozambique, océan Indien). Bulletin de la Société de Pathologie Exotique. 99(2):122128.

Gloria-Soria A, Ayala D, Bheecarry A, Calderon-Arguedas O, Chadee DD, Chiappero M, Coetzee M, Elahee KB, FernandezSalas I, Kamal HA, et al. 2016. Global genetic diversity of Aedes aegypti. Molecular Ecology. 25(21):5377-5395.

Goodman SM. 2008. A new species of Triaenops (Mammalia, Chiroptera) from Aldabra Atoll, Picard Island (Seychelles). Zoosystema. 30 (3):681-693.

Gopaul R. 1995. Surveillance entomologique à Maurice. Cahier Santé. 5:401-405.

Gouagna LC, Dehecq JS, Girod R, Boyer S, Lempérière G, Fontenille D. 2011. Spatial and temporal distribution patterns of Anopheles arabiensis breeding sites in la Reunion Island; multi-year trend analysis of records from 1996-2009. Parasites and Vectors. 4:121-135.

Grjebine A. 1956a. Rapport sommaire sur les moustiques vecteurs de la filariose à l'île Mohéli (archipel des Comores). Rapport IRSM janvier 1956, ORSTOM.

Grjebine A. 1956b. Rapport sommaire sur la première étude des moustiques vecteurs de la filariose des îles Pamandzi et Mayotte. Rapport IRSM 1956, ORSTOM.

Grjebine A. 1966. Insectes diptères Culicidae Anophelinae. Collection Faune de Madagascar n ${ }^{\circ} 2$. Paris: ORSTOM, CNRS; $489 \mathrm{p}$.

Halcrow JG. 1954a. Catalogue of the mosquitoes of Mauritius and Rodrigues. The Mauritius Institute Bulletin, 3:234-248. 
Halcrow JG. 1954b. Occurrence of Culex simpsoni in Mauritius. Nature. 174:466.

Hamon J. 1953. Études biologique et systématique des Culicidae de l'île de la Réunion. Mémoires de l'Institut Scientifique de Madagascar. Série E. 4:521-541.

Harper GA, van Dinther M, Russell JC, Bunbury N. 2015. The response of black rats (Rattus rattus) to evergreen and seasonally arid habitats: Informing eradication planning on a tropical island and impacts on native species. Global Ecology and Conservation. 185:66-74.

Harper JO. 1947. A mosquitoe survey of Mahé, Seychelles. East African Medical Journal. 24(1):25-29.

Hartberg WK, Craig GB Jr. 1968. Reproductive isolation in Stegomyia mosquitoes. I. Sexual isolation between Aedes aegypti and A. mascarensis. Annals of the Entomological Society of America. 61(4):865-870.

Hartberg WK, Craig GB Jr. 1970. Reproductive isolation in Stegomyia mosquitoes. II. Hybrid breakdown between Aedes aegypti and A. mascarensis. Evolution. 24:692-703.

Hermitte LCD. 1931. Occurrence of Anopheles gambiae (costalis) in Aldabra islands (Seychelles). Records of the Malaria Survey of India. 2:643-654

Hopkins GHE. 1952. Mosquitoes of the Ethiopian Region I. Larval bionomics of mosquitoes and taxonomy of Culicinae larvae. 2nd. Ed. With notes and addenda. Mattingly PF, Londres, Royaume-Uni ; 355 p.

Huang YM. 2004. The subgenus Stegomyia of Aedes in the Afrotropical Region with keys to the species (Diptera: Culicidae). Zootaxa. 700:1-120.

Hutson AM. 1981. A preliminary list of insects of Diego Garcia Atoll, Chagos archipelago. Atoll Research Bulletin. 243 ; 129.

Hutson AM. 2004. The bats of Aldabra atoll, Seychelles. Phelsuma. 12:126-132.

Issack MI, Pursem VN, Barkham TMS, Ng LC., Inoue M, Manraj SS. 2010. Reemergence of dengue in Mauritius. Emerging Infectious Diseases. 16:716-718.

Julvez J, Blanchy S. 1988. Le paludisme dans les îles de l'archipel des Comores. Éléments historiques et géophysiques, considérations épidémiologiques. Bulletin de la Société de Pathologie Exotique. 81(5):847-853.

Julvez J, Mouchet J. 1994. Le peuplement culicidien des îles du sud-ouest de l'océan Indien. L'action de l'Homme dans l'importation des espèces d'intérêt médical. Annales de la Société Entomologique de France. 30 (4):391-401.

Jupp PG, McIntosh BM, Blackburna NK. 1986. Experimental assessment of the vector competence of Culex (Culex) neavei Theobald with West Nile and Sindbis viruses in South Africa. Transactions of the Royal Society of Tropical Medicine and Hygiene. 80(2):226-230.

Kirk JB, André JH. 1933. Medical and sanitary notes on Rodrigues. Transactions of the Royal Society of Tropical Medicine and Hygiene. 26(5):449-466.

Lambrecht FL. 1971. Notes on the ecology of Seychelles mosquitoes. Bulletin of Entomological Research. 60:513-532.

Lambrecht FL, van Someren ECC. 1971. Mosquitoes of the Chagos archipelago, Indian Ocean. The Southeast Asian Journal of Tropical Medicine and Public Health. 2(4):483-485.

Lavoipierre R. 1951. Report on a visit to the Chagos Archipelago, Government of Mauritius, 17 October 1951, 2-4.

Le Goff G, Boussès P, Julienne S, Brengues C, Rahola N, Rocamora G, Robert V. 2012. The mosquitoes (Diptera: Culidae) of Seychelles: taxonomy, ecology, vectorial importance, and identification keys. Parasites and Vectors. 5:207-239.

Le Goff G, Brengues C, Robert V. 2013. Stegomyia mosquitoes in Mayotte, taxonomic study and description of Stegomyia pia n. sp. Parasite. 20:31.

Le Goff G, Goodman SM, Elguero E, Robert V. 2014. Survey of the Mosquitoes (Diptera: Culicidae) of Mayotte. PLoS ONE, 9(7): e100696.

Le Goff G, Damiens D, Payet L, Ruttee AH, Jean F, Lebon C, Dehecq JS, Gouagna LC. 2016. Enhancement of the BG-Sentinel trap with varying number of mice for field sampling of male and female Aedes albopictus mosquitoes. Parasites and Vectors. 9(1):514

Manni M, Guglielmino CR, Scolari F, Vega-Rúa A, Failloux AB, Somboon P, Lisa A, Savini G, Bonizzoni M, Gomulski LM, et al. 2017. Genetic evidence for a worldwide chaotic dispersion pattern of the arbovirus vector, Aedes albopictus. PLOS Neglected Tropical Diseases, 30; 11(1): e0005332.

Mac Gregor ME. 1923. Report on the Anophelinae of Mauritius, and on certain aspects of malaria in the colony, with recommendations for a new anti-malaria campaign. London: Colonial Office Publication. Waterlow \& Sons ; 48 p.

Maillard O, Lernout T, Olivier S, Achirafi A, Aubert L, Lepère JF, Thiria J, Pagès F, Filleul L. 2015. Major decrease in malaria transmission on Mayotte Island. Malaria Journal. 14:323.

Mamet JR, Webb-Gebert F. 1980. Insects of medical interest in Mauritius. The Mauritius Institute Bulletin. 9(1):53-73.

Marsden CD, Cornel A, Lee Y, Sanford MR, Norris LC, Goodell PB, Nieman CC, Han S, Rodrigues A, Denis J, et al. 2013. An analysis of two island groups as potential sites for trials of transgenic mosquitoes for malaria control. Evolutionary Applications. 6:706-720.

Mattingly PF. 1963. New and remarkable Aedes (Diptera: Culicidae) from Africa. Proceedings of the Royal Entomological Society of London Series B Taxonomy. 32:165-170.

Mattingly PF, Brown ES. 1955. The mosquitos (Diptera: Culicidae) of the Seychelles. Bulletin of Entomological Research. 46:69-110.

Mattingly PF. 1967. Taxonomy of Aedes aegypti and related species. Bulletin of the World Health Organization. 36:552-554.

Maynard AJ, Ambrose L, Cooper RD, Chow WK, Davis JB, Muzari MO, van den Hurk AF, Hall-Mendelin S, Hasty JM, Burkot TR, et al. 2017. Tiger on the prowl: Invasion history and spatio-temporal genetic structure of the Asian tiger mosquito Aedes albopictus (Skuse 1894) in the Indo-Pacific. PLOS Neglected Tropical Diseases. 11(4): e0005546

McClelland GAH. 1967. Speciation and evolution in Aedes. In: Genetics of insect vectors of diseases. Eds J.W. Wright \& R. Pal. Elsevier, New York \& Amsterdam: 277-311. 
Metselaar D, Grainger CR, Oei KG, Reynolds DG, Pudney M, Leake CJ, Tukei PM, D'Offay RM, Simpson DI. 1980. An outbreak of type 2 dengue fever in the Seychelles, probably transmitted by Aedes albopictus (Skuse). Bulletin of the World Health Organization. 58(6):937-943.

Michot J, Deutsch S. 1977. Les Seychelles, un nucleus sialique. Annales de la Société Géologique de Belgique. 100:147-1.

Mousson L, Dauga C, Garrigues T, Schaffner F, Vazeille M, Failloux AB. 2005. Phylogeography of Aedes (Stegomyia) aegypti (L.) and Aedes (Stegomyia) albopictus (Skuse) (Diptera: Culicidae) based on mitochondrial DNA variations. Genetical Research. 86(1):1-11.

Ottino P. 1974. Le Moyen-âge de l'océan Indien et le peuplement de Madagascar in Annuaire des pays de l'océan Indien, vol 1, Presse Universitaires d'Aix-Marseille, Aix-les-Bains, pp. 197-221.

Paulian R. 1950. L'île Europa, une dépendance de Madagascar. Le Naturaliste Malgache, 2:77-86.

Paulian R. 1961. La zoogéographie de Madagascar et des îles voisines. Collection Faune de Madagascar, $n^{\circ} 13$, Institut de Recherche Scientifique, Tananarive ; $515 \mathrm{p}$.

Paulian R. 1964. Composition et origine de l'entomofaune comorienne. Bulletin de la Société Entomologique de France. 69:167-173.

Petrarca V, Sabatinelli G, Di Deco MA, Papakay M. 1990. The Anopheles gambiae complex in the Federal Islamic Republic of Comoros (Indian Ocean): some cytogenetic and biometric data. Parassitologia. 32(3):371-380.

Poisson H. 1923. Rapport de tournée à l'Ile Europa, et retour par terre, via Manombo (du 16 au 26 février 1923). Bulletin Economique de Madagascar, Tananarive. 131-141.

Prod'hon J. 1972. Etude parasitologique de la filariose de Bancroft à Anjouan. Cahiers ORSTOM Série Entomologie Médicale et Parasitologie. 10(3): 263-273.

Ravaonjanahary C, Brunhes J. 1977. Un nouvel Aedes du genre Skusea découvert à Madagascar : Aedes (Skusea) moucheti sp. n. Cahiers ORSTOM Série Entomologie Médicale et Parasitologie. 15(3): 213-215.

Reinert JF, Harbach RE, Kitching IJ. 2009. Phylogeny and classification of tribe Aedini (Diptera: Culicidae. Zoological Journal of the Linnean Society. 157:700-794.

Ribeiro E, da Cunha Ramos H. 1980. Research on the mosquitoes of Angola (Diptera, Culicidae) X The genus Culex L., 1758. Check-list with new records, keys to females and larvae, distribution, and taxonomic and bioecological notes. Junta de investigacoes cientificas do ultramar. Estudos, Ensaios e documentos. $134 ; 175$ p.

Robert V. 2003. Rapport d'une mission entomologique à Mohéli (Union des Comores) du 26 au 29 février 2003. Documents Techniques de l'Institut Pasteur de Madagascar - Entomologie. 03 ; 6 p.

Robert V, Rocamora G, Juliennes S, Goodman SM. 2011. Why are anopheline mosquitoes not present in the Seychelles? Malaria Journal. 10:31.

Rogo LMN, White GB, Odhiambo RC. 1985. Salinity relationships of mosquitoes breeding in a brackish pond on the Kenya coast. International Journal of Tropical Insect Science. 6(1):91-95.

Russell JC, le Corre M. 2009. Introduced mammal impacts on seabirds in the îles Éparses, Western Indian Ocean. Marine Ornithology. 37:121-129.

Sabatinelli G, Ranieri E, Gianzi FP, Papakay M, Cancrini G. 1994. Rôle de Culex quinquefasciatus dans la transmission de la filariose de Bancroft dans la république Fédérale Islamique des Comores (Océan Indien). Parasite. 1:71-76.

Salvan M, Mouchet J. 1994. Aedes albopictus et Aedes aegypti à l'île de la Réunion. Annales de la Société Belge de Médecine Tropicale. 74 (4):323-326.

Sanchez M. 2015. Outil de gestion pour les reptiles terrestres de l'île de Juan de Nova (Océan Indien, Canal du Mozambique): inventaire, distribution et densités des populations pré-éradication des chats. Rapport Nature Océan Indien. 43 pp + annexes.

Sang RC, Ahmed O, Faye O, Kelly CLH., Yahaya AA, Mmadi I, Toilibou A, Sergon K, Brown J, Agata N, et al. 2008. Entomologic investigations of a chikungunya virus epidemic in the Union of the Comoros, 2005. The American Journal of Tropical Medicine and Hygiene. 78(1):77-82.

Seabrook W. 1990. The impact of the feral cat (Felis catus) on the native fauna of Aldabra atoll, Seychelles. Revue d'Ecologie (Terre Vie). 45(2):135-145.

Service MW. 1997. Mosquito (Diptera: Culicidae) Dispersal. The Long and Short of It. Journal of Medical Entomology. 34(6):579-588.

Siddall M, Rohling EJ, Almogi-Labin A, Hemleben C, Meischner D, Schmelzer I, Smeed DA. 2003. Sea-level fluctuations during the last glacial cycle. Nature. 423:853-858.

Subra R. 1975. Urbanisation et filariose de Bancroft en Afrique et à Madagascar. Cahiers ORSTOM Série Entomologie Médicale et Parasitologie. 13:193-203.

Tantely ML, Le Goff G, Boyer S, Fontenille D. 2016. An updated checklist of mosquito species (Diptera: Culicidae) from Madagascar. Parasite. 23:20.

Theobald FV. 1912. Reports of the Percy Sladen Trust Expedition to the Indian Ocean in 1905. No. V.- Diptera, Culicidae. Transactions of the Linnean Society of London. 15:81-94.

van Someren ECC. 1947. The description of a new mosquito from the Seychelles. East African Medical Journal. 24:29-35.

van Someren ECC. 1971. The description of a new Aedes mosquito of the subgenus Skusea from the Seychelles. Journal of Entomology Series B, Taxonomy. 40:21-25.

van Someren ECC. 1972. On the Status of Aedes (Ochlerotatus) fryeri (Theobald) and Aedes (Ochlerotatus) mombasaensis Mattingly. Mosquito Systematic. (4)3:90.

Viette P. 1966. L'entomofaune de l'île Europa. In: Legendre R (Ed), Mission scientifique à l'île Europa. Muséum National d'Histoire Naturelle (Série A-Zoologie), Paris. 41:191-210.

WHO 2012. Eliminating malaria. Case-study 4. Preventing reintroduction in Mauritius. Who Library, Geneva ; 75 p. 
Wilkerson RC, Linton YM, Fonseca DM, Schultz TR, Price DC, Strickman DA. 2015. Making mosquito taxonomy useful: a stable classification of tribe Aedini that balances utility with current knowledge of evolutionary relationships. PLoS ONE, 10(7): e0133602.

Willaine P. 1984. Les sols de l'île Maurice. Mauritius Sugar Industry Research Institute, Moka \& ORSTOM, Paris ; 184 p.

Yersin C, Bovet P, Herminie P, Zeller H. 1999. Public health importance of mosquito-borne diseases in the Seychelles (Indian Ocean). The Infectious Disease Review. 1(3):189-199.

Zeller HG. 1998. Dengue, arbovirus et migration dans l'Océan Indien. Bulletin de la Société de Pathologie Exotique. 91(1):5660. 


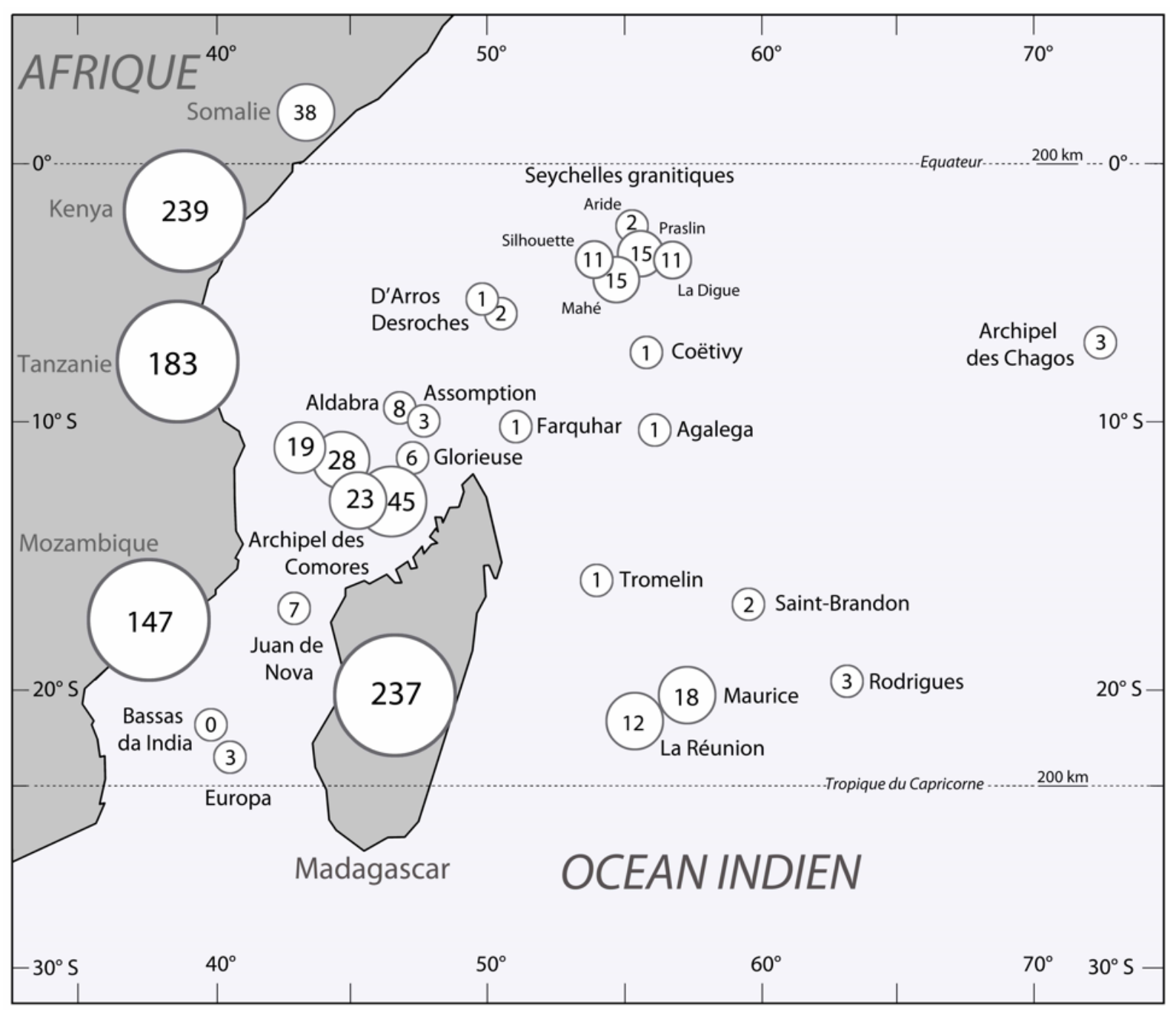

Figure 1. Localisation schématique des diverses îles du sud-ouest de l'océan Indien, avec indication du nombre d'espèces culicidiennes. Les chiffres des pays africains sont calculés à partir des données de la base WRBU (Smithsonian Institution, Washington, USA) et de la base ARIM (Institut de Recherche pour le Développement, Montpellier, France). 


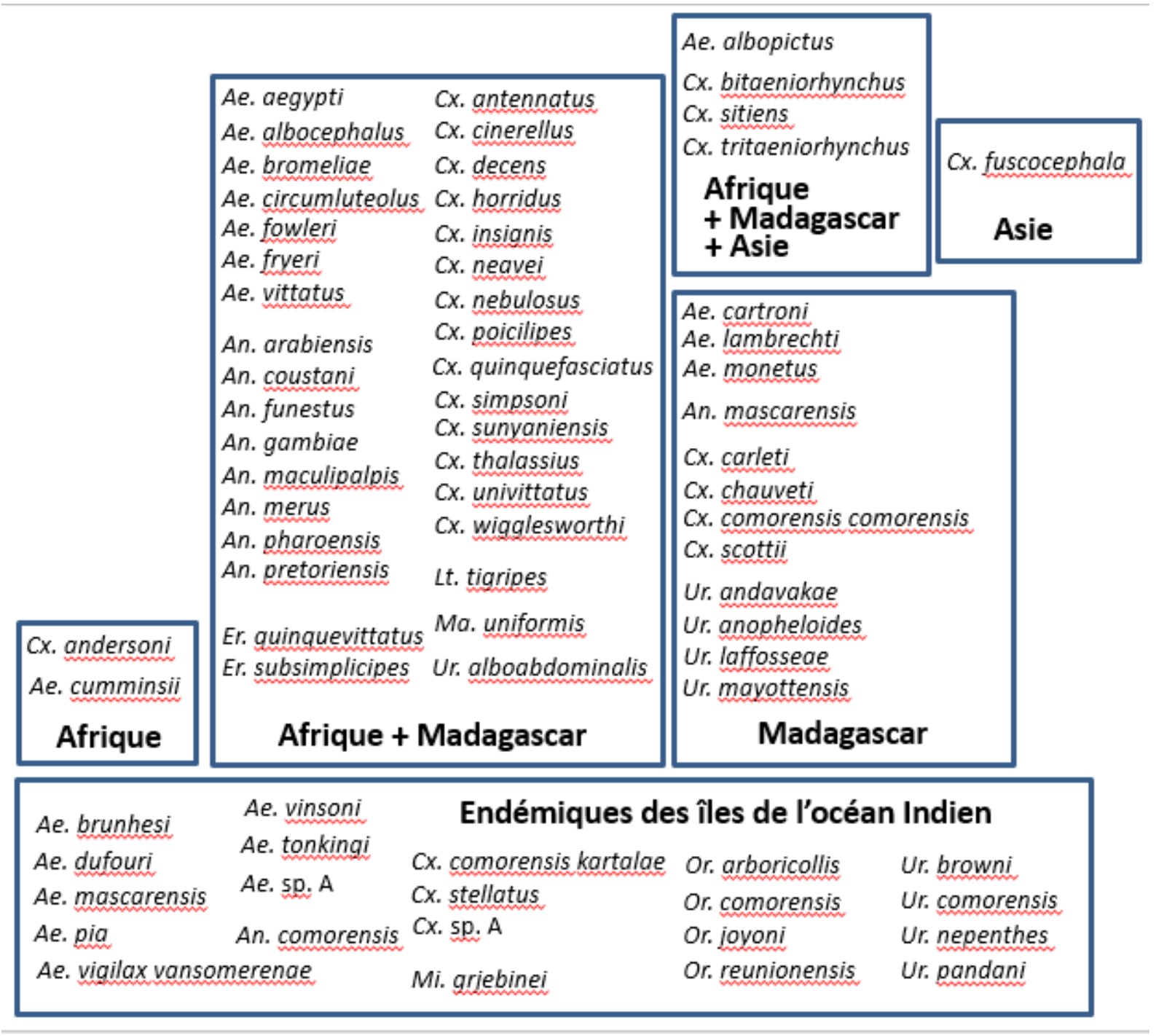

Figure 2. Affinités bio-géographiques des espèces culicidiennes présentes dans les îles du sud-ouest de l'océan Indien. À noter que Culex comorensis, ici mentionnée deux fois, $C x . c$. comorensis et $C x . c$. kartalae, n'est pas comptabilisée comme endémique de ces îles. 
Tableau 1. Synonymies taxonomiques des moustiques de la tribu des Aedini distribués dans le sud-ouest de l'océan Indien.

\begin{tabular}{ll}
$\begin{array}{c}\text { Nomenclature binomiale recommandée } \\
\text { par Wilkerson et al, 2015 } \\
\text { et utilisée dans le présent article }\end{array}$ & \multicolumn{1}{c}{$\begin{array}{c}\text { Nomenclature binomiale } \\
\text { dans le site www.mosquito-taxonomic- } \\
\text { inventory* }\end{array}$} \\
\hline Aedes (Adm.) cumminsii (Theobald, 1903) & $\begin{array}{l}\text { Aedimorphus cumminsii } \\
\text { Aedes (Adm.) fowleri (de Charmoy, 1908) }\end{array}$ \\
Aedes (Adm.) sp. A & $\begin{array}{l}\text { Aedimorphus fowleri } \\
\text { Aedimorphus sp. A*** }\end{array}$ \\
Aedes (Coe.) fryeri (Theobald, 1912) & Ochlerotatus (Coetzeemyia) fryeri \\
Aedes (Fre.) vittatus (Bigot, 1861) & Fredwardsius vittatus \\
Aedes (Muc.) tonkingi Gebert, 1948 & Mucidus (Mucidus) tonkingi \\
Aedes (Neo.) circumluteolus (Theobald, 1908) & Neomelaniconion circumluteolus \\
Aedes (Och.) dufouri (Hamon, 1953) & Ochlerotatus dufouri \\
Aedes (Och.) vigilax vansomerenae Mattingly, 1955 & Ochlerotatus vigilax vansomerenae \\
Aedes (Pmt.) albocephalus (Theobald, 1903) & Polyleptiomyia albocephala \\
Aedes (Sku.) cartroni (Ventrillon, 1906) & Skusea cartroni \\
Aedes (Sku.) lambrechti Van Someren, 1971 & Skusea lambrechti \\
Aedes (Stg.) aegypti (Linnaeus, 1762) & Stegomyia (Stegomyia) aegypti \\
Aedes (Stg.) albopictus (Skuse, 1895) & Stegomyia albopicta \\
Aedes (Stg.) bromeliae (Theobald, 1911) & Stegomyia (Mukwaya) bromeliae \\
Aedes (Stg.) mascarensis MacGregor, 1924 & Stegomyia (Stegomyia) mascarensis \\
Aedes (Stg.) pia (Le Goff \& Robert, 2013) & Stegomyia (Stegomyia) pia \\
Aedes (Stg.) vinsoni Mattingly, 1953 & Stegomyia vinsoni \\
Aedes (Zav.) brunhesi Reinert, 1999 & Zavortinkius brunhesi \\
Aedes (Zav.) monetus Edwards, 1935 & Zavortinkius monetus \\
\hline
\end{tabular}

* Consultation le 22 octobre 2017

*** in Le Goff et al (2014) 
Tableau 2. Liste des genres, sous-genres et espèces de moustiques (Diptera : Culicidae) de l'archipel des Comores.

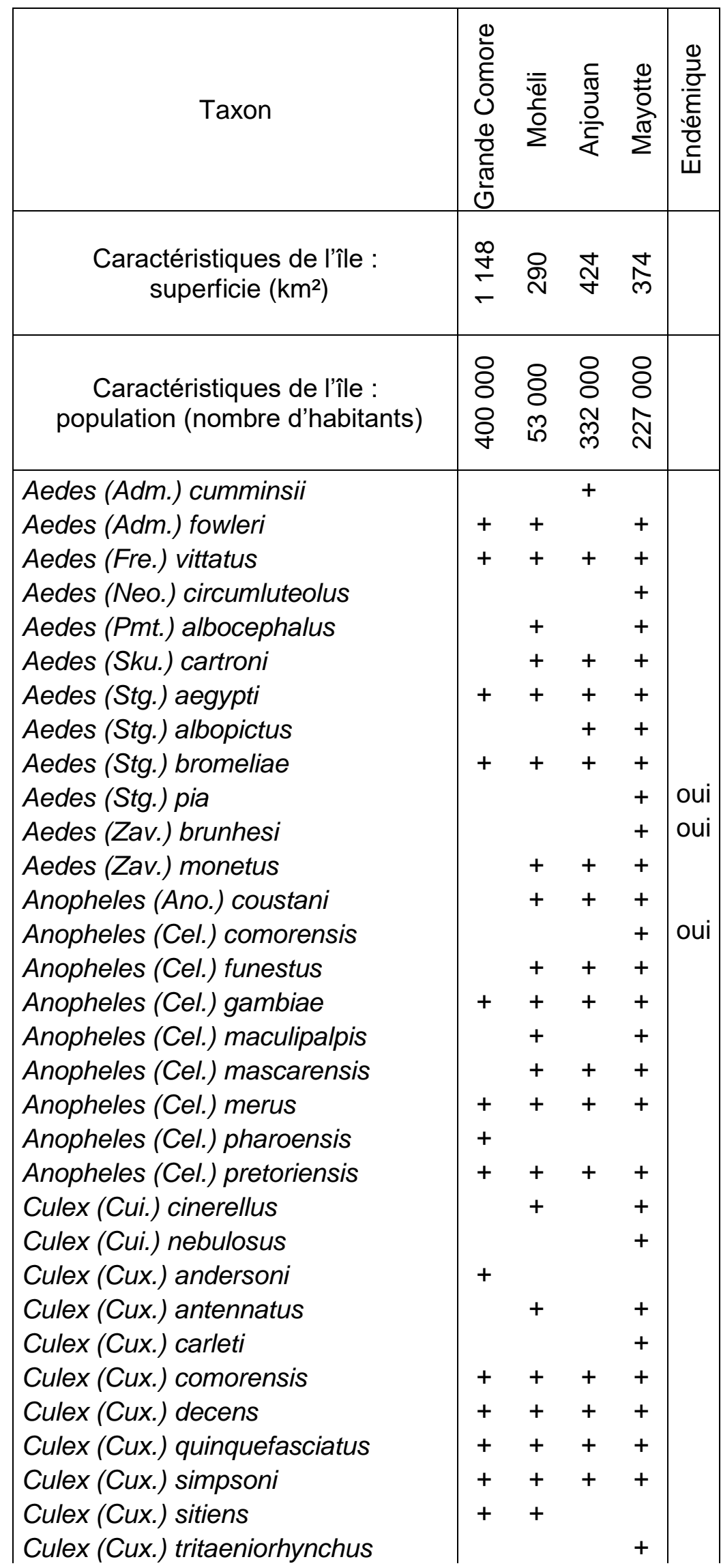


Culex (Cux.) univittatus

Culex (Eum.) chauveti

Culex (Eum.) horridus

Culex (Eum.) sunyaniensis

Culex (Eum.) wigglesworthi

Culex (Ocu.) bitaeniorhynchus

Culex sp. A

Eretmapodites quinquevittatus

Eretmapodites subsimplicipes

Lutzia (Mlt.) tigripes

Mansonia (Mnd.) uniformis

Mimomyia (Ing.) grjebinei

Orthopodomyia comorensis

Orthopodomyia joyoni

Uranotaenia (PfC.) comorensis

Uranotaenia (PfC.) laffosseae

Uranotaenia (Ura.) alboabdominalis

Uranotaenia (Ura.) andavakae

Uranotaenia (Ura.) anopheloides

Uranotaenia (Ura.) mayottensis

Total $=52$

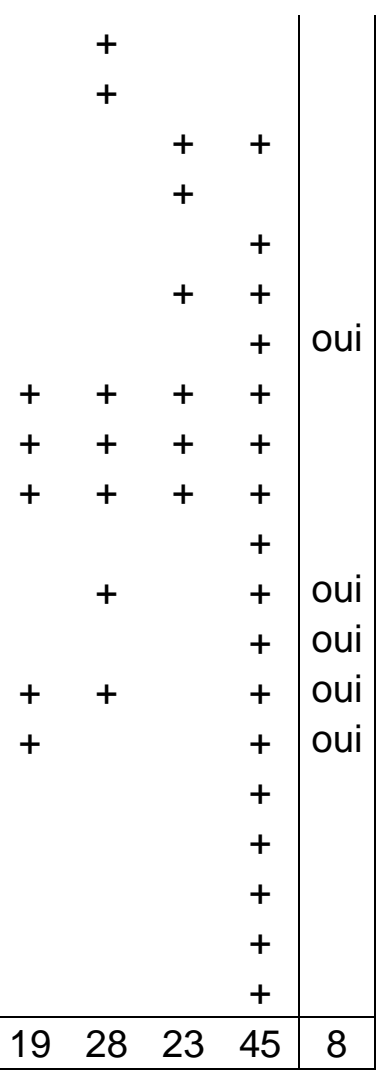


Tableau 3. Liste des genres, sous-genres et espèces de moustiques (Diptera : Culicidae) des lles Eparses de l'océan Indien.

\begin{tabular}{|c|c|c|c|c|c|c|}
\hline Taxon & 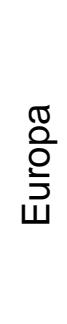 & 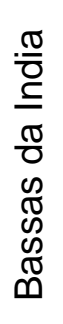 & $\begin{array}{l}\stackrel{\pi}{0} \\
0 \\
2 \\
0 \\
0 \\
\frac{1}{0} \\
\stackrel{0}{7}\end{array}$ & 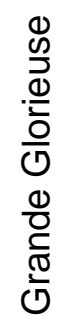 & 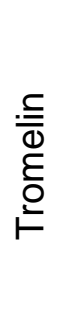 & 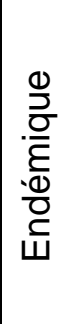 \\
\hline $\begin{array}{c}\text { Caractéristiques de l'île : } \\
\text { superficie }\left(\mathrm{km}^{2}\right)\end{array}$ & 28 & 1 & 5 & 7 & 1 & \\
\hline $\begin{array}{c}\text { Caractéristiques de l'île : } \\
\text { population (nombre d'habitants) }\end{array}$ & 0 & 0 & 0 & 0 & 0 & \\
\hline $\begin{array}{l}\text { Aedes (Coe.) fryeri } \\
\text { Aedes (Stg.) aegypti } \\
\text { Aedes (Stg.) albopictus } \\
\text { Anopheles (Cel.) gambiae s.l. } \\
\text { Culex (Cux.) quinquefasciatus } \\
\text { Culex (Cux.) sitiens } \\
\text { Culex (Cux.) tritaeniorhynchus } \\
\text { Lutzia (Mlt.) tigripes } \\
\text { Mansonia (Mnd.) uniformis }\end{array}$ & $\begin{array}{l}+ \\
+\end{array}$ & & $\begin{array}{l}+ \\
+ \\
+ \\
+ \\
+ \\
+ \\
+ \\
+\end{array}$ & $\begin{array}{l}+ \\
+ \\
+ \\
+ \\
+ \\
+\end{array}$ & + & \\
\hline Total $=9$ & 3 & 0 & 7 & 6 & 1 & 0 \\
\hline
\end{tabular}


Tableau 4. Liste des genres, sous-genres et espèces de moustiques (Diptera : Culicidae) des Mascareignes.

\begin{tabular}{|c|c|c|c|c|c|c|}
\hline Taxon & 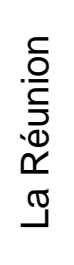 & 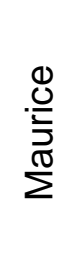 & 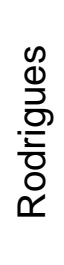 & 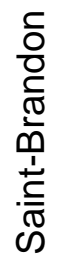 & $\frac{\sqrt[\sigma]{0}}{\frac{\mathbb{d}}{\widetilde{\sigma}}}$ & 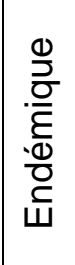 \\
\hline $\begin{array}{c}\text { Caractéristiques de l'île : } \\
\text { superficie }\left(\mathrm{km}^{2}\right)\end{array}$ & $\frac{\sim}{\stackrel{N}{N}}$ & $\begin{array}{l}\underbrace{}_{0} \\
\infty\end{array}$ & 응 & $\stackrel{m}{r}$ & $\stackrel{d}{\sim}$ & \\
\hline $\begin{array}{c}\text { Caractéristiques de l'île: } \\
\text { population (nombre d'habitants) }\end{array}$ & $\begin{array}{l}\text { ○ } \\
\circ \\
\circ \\
\text { ○ }\end{array}$ & $\begin{array}{l}8 \\
8 \\
8 \\
\stackrel{N}{ } \\
-\end{array}$ & $\frac{8}{8}$ & 8 & ஓి & \\
\hline $\begin{array}{l}\text { Aedes (Adm.) fowleri } \\
\text { Aedes (Muc.) tonkingi } \\
\text { Aedes (Och.) dufouri } \\
\text { Aedes (Stg.) aegypti } \\
\text { Aedes (Stg.) albopictus } \\
\text { Aedes (Stg.) mascarensis } \\
\text { Aedes (Stg.) vinsoni } \\
\text { Anopheles (Ano.) coustani } \\
\text { Anopheles (Cel.) arabiensis } \\
\text { Anopheles (Cel.) maculipalpis } \\
\text { Anopheles (Cel.) merus } \\
\text { Culex (Cux.) neavei } \\
\text { Culex (Cux.) poicilipes } \\
\text { Culex (Cux.) quinquefasciatus } \\
\text { Culex (Cux.) simpsoni } \\
\text { Culex (Cux.) thalassius } \\
\text { Culex (Cux.) tritaeniorhynchus } \\
\text { Culex (Cux.) univittatus } \\
\text { Culex (Eum.) insignis } \\
\text { Lutzia (Mlt.) tigripes } \\
\text { Orthopodomyia arboricollis } \\
\text { Orthopodomyia reunionensis }\end{array}$ & $\begin{array}{l}+ \\
+ \\
+ \\
+\end{array}$ & $\begin{array}{l}+ \\
+ \\
+ \\
+ \\
+ \\
+ \\
+ \\
+ \\
+ \\
+ \\
+ \\
+ \\
+ \\
+ \\
+ \\
+ \\
+ \\
+\end{array}$ & $\begin{array}{l}+ \\
+\end{array}$ & $\begin{array}{l}+ \\
+\end{array}$ & + & $\begin{array}{l}\text { oui } \\
\text { oui }\end{array}$ \\
\hline Total $=22$ & 12 & 18 & 3 & 2 & 1 & 6 \\
\hline
\end{tabular}


Tableau 5. Liste des genres, sous-genres et espèces de moustiques (Diptera: Culicidae) des Seychelles granitiques.

\begin{tabular}{|c|c|c|c|c|c|c|}
\hline Taxon & $\frac{\mathscr{Q}}{\frac{\mathscr{N}}{\tilde{N}}}$ & 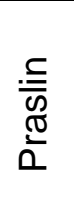 & $\begin{array}{l}\frac{0}{\partial} \\
\stackrel{0}{0} \\
\text { 똑 }\end{array}$ & 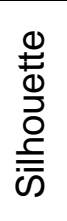 & $\frac{0}{\frac{0}{2}}$ & 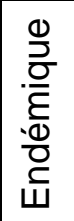 \\
\hline $\begin{array}{c}\text { Caractéristiques de l'île : } \\
\text { superficie }\left(\mathrm{km}^{2}\right)\end{array}$ & $\stackrel{\&}{\leftarrow}$ & $\stackrel{0}{\infty}$ & $\begin{array}{l}\infty \\
\sigma^{-1}\end{array}$ & ㅇ & $\hat{0}$ & \\
\hline $\begin{array}{c}\text { Caractéristiques de l'île : } \\
\text { population (nombre d'habitants) }\end{array}$ & 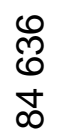 & $\begin{array}{l}\mathscr{1} \\
\stackrel{م}{ } \\
\infty\end{array}$ & 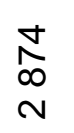 & $\stackrel{\llcorner}{m}$ & 0 & \\
\hline $\begin{array}{l}\text { Aedes (Och.) vigilax vansomerenae } \\
\text { Aedes (Pmt.) albocephalus } \\
\text { Aedes (Sku.) lambrechti } \\
\text { Aedes (Stg.) aegypti } \\
\text { Aedes (Stg.) albopictus } \\
\text { Culex (Cux.) fuscocephala } \\
\text { Culex (Cux.) quinquefasciatus } \\
\text { Culex (Cux.) scottii } \\
\text { Culex (Cux.) simpsoni } \\
\text { Culex (Cux.) tritaeniorhynchus } \\
\text { Culex (Eum.) stellatus } \\
\text { Culex (Eum.) sunyaniensis } \\
\text { Culex (Eum.) wigglesworthi } \\
\text { Mansonia (Mnd.) uniformis } \\
\text { Uranotaenia (Pfc.) browni } \\
\text { Uranotaenia (Pfc.) nepenthes } \\
\text { Uranotaenia (Pfc.) pandani }\end{array}$ & $\begin{array}{l}+ \\
+ \\
+ \\
+ \\
+ \\
+ \\
+ \\
+ \\
+ \\
+ \\
+\end{array}$ & $\begin{array}{l}+ \\
+ \\
+ \\
+ \\
+ \\
+ \\
+ \\
+ \\
+ \\
+ \\
+ \\
+\end{array}$ & $\begin{array}{l}+ \\
+ \\
+ \\
+ \\
+\end{array}$ & $\begin{array}{l}+ \\
+ \\
+\end{array}$ & + & $\begin{array}{l}\text { oui } \\
\text { oui } \\
\text { oui }\end{array}$ \\
\hline Total $=17$ & 15 & 15 & 11 & 10 & 2 & 5 \\
\hline
\end{tabular}


Tableau 6. Liste des genres, sous-genres et espèces de moustiques (Diptera : Culicidae) des Seychelles coralliennes et des Chagos.

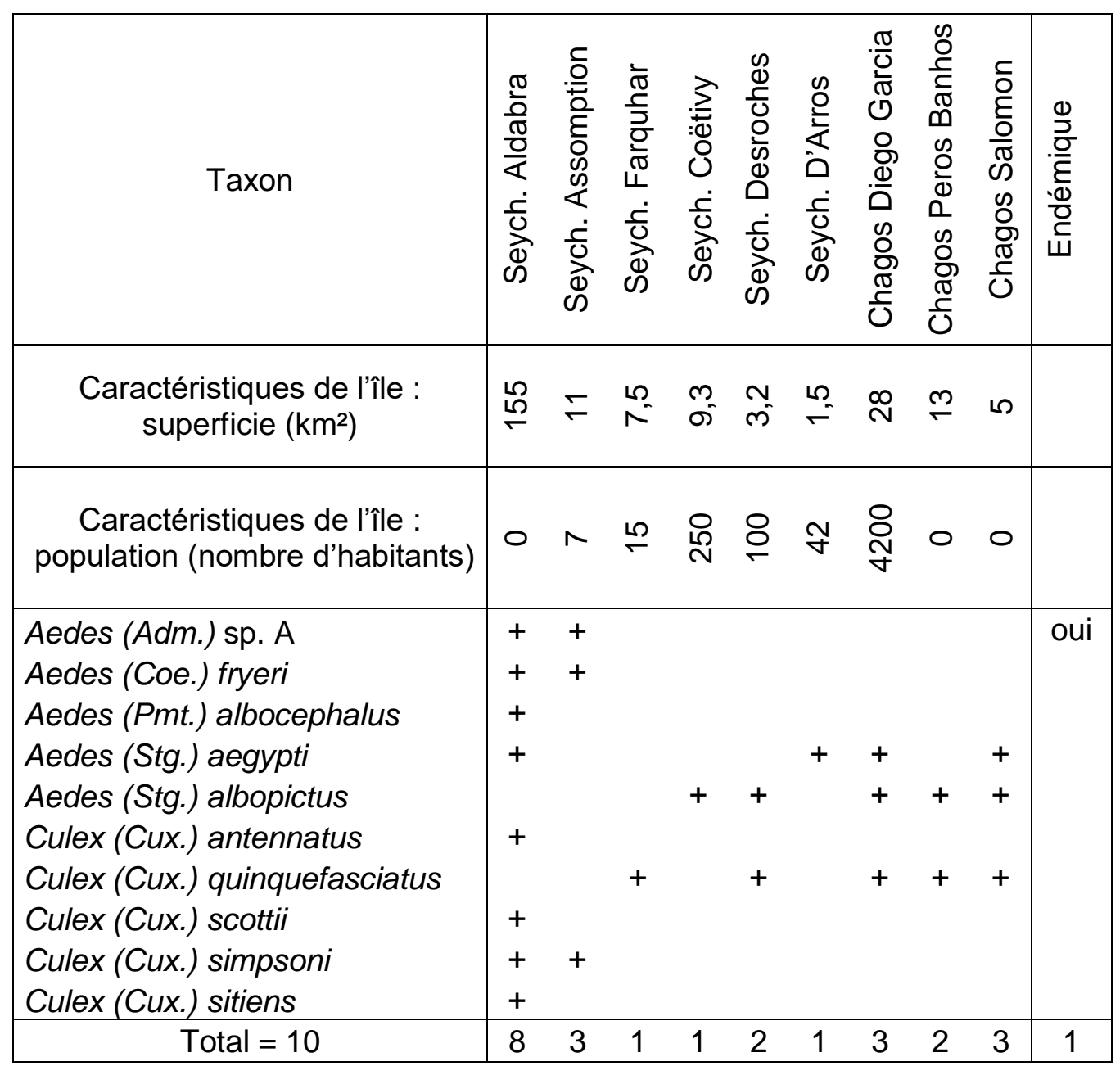


Tableau S1. Liste et distribution par île des 73 espèces de moustiques des îles du sud-ouest de l'océan Indien (feuille 1). Source bibliographique de la présence de chaque espèce culicidienne dans chaque île (feuille 2). Fichier xls.

Tableau S2. Liste et distribution par groupe d'îles des 73 espèces de moustiques des îles du sud-ouest de l'océan Indien, avec mention de leur éventuel caractère endémique. Fichier xls. 